\title{
GRADED INDUCTION FOR SPECHT MODULES
}

\author{
JUN HU AND ANDREW MATHAS
}

\begin{abstract}
Recently Brundan, Kleshchev and Wang introduced a $\mathbb{Z}$-grading on the Specht modules of the degenerate and non-degenerate cyclotomic Hecke algebras of type $G(\ell, 1, n)$. In this paper we show that induced Specht modules have an explicit filtration by shifts of graded Specht modules. This proves a conjecture of Brundan, Kleshchev and Wang.
\end{abstract}

\section{INTRODUCTION}

In a remarkable series of papers Brundan and Kleshchev (and Wang) [6 $[\mathbf{8}]$ have shown that the cyclotomic Hecke algebras of type $G(\ell, 1, n)$ are $\mathbb{Z}$-graded algebras and they have proved a graded analogue of Ariki's categorification theorem [1]. This work builds upon the work of Khovanov and Lauda [16, §3.4] and Rouquier [25]'s introduction of the quiver Hecke algebras which are certain graded algebras which categorify the negative part of quantum group of an arbitrary Kac-Moody Lie algebra.

The representation theory of the cyclotomic Hecke algebras of type $G(\ell, 1, n)$ is very well developed with the Specht modules introduced in [3, 10] playing a central role. For each multipartition $\boldsymbol{\mu}$ Brundan, Kleshchev and Wang [8] introduced a $\mathbb{Z}$-grading on each Specht module $S^{\boldsymbol{\mu}}$ which is isomorphic to the (ungraded) Specht module upon forgetting the grading. In [8, Theorem 4.11] they showed that restriction of the graded Specht module has a graded Specht filtration. By Frobenius reciprocity in the Grothendieck group there is an analogous formula for the induced graded Specht modules. Brundan, Kleshchev and Wang conjectured that this should correspond to a filtration of the induced Specht module by shifts of graded Specht modules. In this paper we prove this conjecture.

To state our main results, fix an integral domain $R$ and an integer $e \in\{0,2,3,4, \ldots\}$ such that either $e=0$ or $e$ is invertible in $R$ whenever $e$ is not prime, let $\mathscr{H}_{n}^{\Lambda}$ be the graded cyclotomic quiver Hecke algebra (over $R$ ) determined by $e$ and the dominant weight $\Lambda$ (see Definition 3.1). The algebras $\mathscr{H}_{n}^{\Lambda}$ include the cyclotomic Hecke algebras of type $G(\ell, 1, n)$ as special cases. When $R$ is a field there is a natural graded embedding $\mathscr{H}_{n} \Lambda \hookrightarrow$ $\mathscr{H}_{n+1}^{\Lambda}$ which makes $\mathscr{H}_{n+1}^{\Lambda}$ into a free $\mathscr{H}_{n}^{\Lambda}$-module by the main theorem of [13]. There is an induction functor

$$
\text { Ind }: \operatorname{Mod}-\mathscr{H}_{n}^{\Lambda} \longrightarrow \operatorname{Mod}-\mathscr{H}_{n+1}^{\Lambda} ; M \mapsto M \otimes_{\mathscr{H}_{n}^{\Lambda}} \mathscr{H}_{n+1}^{\Lambda} \text {. }
$$

By projecting onto the blocks of $\mathscr{H}_{n+1}^{\Lambda}$ the induction functor decomposes as

$$
\text { Ind }=\bigoplus_{i \in I} i \text {-Ind }
$$

where $I=\mathbb{Z} / e \mathbb{Z}$.

2000 Mathematics Subject Classification. 20C08, 20C30, 05E10.

Key words and phrases. Cyclotomic Hecke algebras, Khovanov-Lauda-Rouquier algebras, cellular algebras.

This research was supported, in part, by the Australian Research Council. 
Fix $i \in I$ and let $\boldsymbol{\mu}$ be a multipartition of $n$. Let $\boldsymbol{\alpha}_{1}, \ldots, \boldsymbol{\alpha}_{z}$ be the multipartitions of $n+1$ obtained by adding an addable $i$-node to $\boldsymbol{\mu}$ (see before Definition 3.3), ordered so that $\boldsymbol{\alpha}_{1} \triangleright \cdots \triangleright \boldsymbol{\alpha}_{z}$, where $\unrhd$ is the dominance order on multipartitions (see $\S 2.1$ ). Given an addable node $A$ of $\boldsymbol{\mu}$ then we define the integer $d_{A}(\boldsymbol{\mu})$ in Definition 3.3 below. Finally, if $M$ is a graded $\mathscr{H}_{n}^{\Lambda}$-module and $d \in \mathbb{Z}$ then $M\langle d\rangle$ is the graded $\mathscr{H}_{n}^{\Lambda}$-module obtained by shifting the grading on $M$ by $d$; see Section 4.

Main Theorem. Suppose that $R$ is an integral domain and that either $e=0$ or $e$ is invertible in $R$ whenever $e$ is not prime. Let $\boldsymbol{\mu} \in \mathscr{P}_{n}$ and $i \in I$. Then the induced graded Specht module $i$-Ind $S^{\mu}$ has a graded Specht filtration. That is, there exists a filtration

$$
0=I_{0} \subset I_{1} \subset \cdots \subset I_{z}=i \text {-Ind } S^{\mu},
$$

such that $I_{j} / I_{j-1} \cong S^{\boldsymbol{\alpha}_{j}}\left\langle d_{A_{j}}(\boldsymbol{\mu})\right\rangle$.

Our arguments easily extend to the analogous result for the induced graded dual Specht modules $S_{\mu}$; see Corollary 4.6 .

When $e=0$ and $\ell=2$ this result can be deduced from [9. Lemma 3.4]. In the ungraded setting, when $\mathscr{H}_{n}^{\Lambda}$ can be identified with the cyclotomic Hecke algebras of type $G(\ell, 1, n)$, this result was established by the second author [22]. The main observation in [22] is that the filtration of the induced Specht module is the restriction of a Specht filtration of a closely related family of 'induced' modules $M(\boldsymbol{\mu})$. In general, we do not know how to construct graded lifts of the modules $M(\boldsymbol{\mu})$ so we cannot use this approach here.

To prove our Main Theorem we instead use a beautiful construction of Ryom-Hansen which gives an explicit filtration of the induced Specht modules for the Hecke algebras of the symmetric group [26] - in the ungraded setting Ryom-Hansen gave the first proof of our Main Theorem for the Hecke algebras of the symmetric groups. In order to adapt Ryom-Hansen's construction to the graded setting we use our recent construction of a homogeneous cellular basis for $\mathscr{H}_{n}^{\Lambda}$ and our realization, up to shift, of the graded Specht modules as graded submodules of $\mathscr{H}_{n}^{\Lambda}$; see [13].

The outline of this paper is as follows. In the Chapter 2 we recall the results that we need from the ungraded representation theory of the Hecke algebras of type $G(\ell, 1, n)$ and use this to establish a strong result (Theorem 2.12), about the transition matrices between the standard and seminormal bases of these algebras in the semisimple case. In Chapter 3 we prove an analogous result (Theorem 3.7), for the transition matrices between the standard and homogeneous bases of the graded algebras. One consequence of these results is a necessary condition for the products $m_{\mathfrak{s t}} n_{\mathfrak{u v}}$ and $\psi_{\mathfrak{s t}} \psi_{\mathfrak{u v}}^{\prime}$ to be non-zero, where $\left\{m_{\mathfrak{s t}}\right\}$ and $\left\{n_{\mathfrak{u v}}\right\}$ are the standard bases corresponding to the trivial and sign representations of $\mathscr{H}_{n}$ and $\left\{\psi_{\mathfrak{s t}}\right\}$ and $\left\{\psi_{\mathfrak{u v}}^{\prime}\right\}$ are homogeneous analogues of these bases. In Chapter 4 we use this non-vanishing condition, together with the ideas of Ryom-Hansen [26], to prove our Main Theorem.

\section{Cyclotomic Hecke algebras And strong dominance}

The aim of this paper is to understand the effect of the graded induction functors on the Specht modules. To do this we first need to prove a strong result about certain structure constants in the ungraded case.

2.1. Cyclotomic Hecke algebras. We start with the definition of the cyclotomic Hecke algebras of type $G(\ell, 1, n)$.

Fix an integral domain $R$ and an integer $\ell \geq 1$. Define $\delta_{\xi 1}=1$ if $\xi=1$ and $\delta_{\xi 1}=0$ otherwise. 
2.1. Definition. Suppose that $\xi \in R$ is invertible and that $\mathbf{Q}=\left(Q_{1}, \ldots, Q_{\ell}\right) \in R^{\ell}$. The cyclotomic Hecke algebra $\mathscr{H}_{n}(\xi, \mathbf{Q})=\mathscr{H}_{n}^{R}(\xi, \mathbf{Q})$ of type $G(\ell, 1, n)$ and with parameters $\xi$ and $\mathbf{Q}$ is the unital associative $R$-algebra with generators $L_{1}, \ldots, L_{n}, T_{1}, \ldots, T_{n-1}$ and relations

$$
\begin{array}{rlrl}
\left(L_{1}-Q_{1}\right) \ldots\left(L_{1}-Q_{\ell}\right) & =0, & L_{r} L_{t}=L_{t} L_{r}, \\
\left(T_{r}+1\right)\left(T_{r}-\xi\right) & =0, & T_{r} L_{r}+\delta_{\xi 1}=L_{r+1}\left(T_{r}-\xi+1\right), \\
T_{s} T_{s+1} T_{s} & =T_{s+1} T_{s} T_{s+1}, & & \\
T_{r} L_{t} & =L_{t} T_{r}, & & \text { if } t \neq r, r+1, \\
T_{r} T_{s} & =T_{s} T_{r}, & & \text { if }|r-s|>1,
\end{array}
$$

where $1 \leq r<n, 1 \leq s<n-1$ and $1 \leq t \leq n$.

This definition, which we used in [13], allows us to simultaneously treat the degenerate and non-degenerate cyclotomic Hecke algebras of type $G(\ell, 1, n)$. By the Morita equivalence reductions of [11, Theorem 1.1] and [5, Theorem 5.19] it is enough to consider the cases where the parameters $\mathbf{Q}$ are integral in the sense that each $Q_{s}$ is an integral power of $\xi$, if $\xi \neq 1$, and $\mathbf{Q} \in \mathbb{Z}^{\ell}$ if $\xi=1$.

Define the quantum characteristic of $\xi$ to be the smallest positive integer $e$ such that $1+\xi+\cdots+\xi^{e-1}=0$; or 0 if no such positive integer exists. Then $e \in\{0,2,3,4, \ldots\}$. We fix a multicharge $\boldsymbol{\kappa}=\left(\kappa_{1}, \ldots, \kappa_{\ell}\right) \in \mathbb{Z}^{\ell}$ such

a) if $e \neq 0$ then $\kappa_{l}-\kappa_{l+1} \geq n$ for $1 \leq l<\ell$.

b) if $\xi=1$ then $Q_{l} \equiv \kappa_{l}(\bmod e)$, for $1 \leq l \leq \ell$.

c) if $\xi \neq 1$ then $Q_{l}=\xi^{\kappa_{l}}$, for $1 \leq l \leq \ell$.

This choice of multicharge plays a role in what follows only in helping us make a good choice of modular system as in [13, §4.2]. The multicharge $\kappa$ determines the parameters Q. Moreover, for a fixed choice of multicharge, $\mathscr{H}_{n}$ depends only on the quantum characteristic $e$ of $\xi$, and not on the choice of $\xi$ itself, by [6. Theorem 6.1]. Therefore, we write $\mathscr{H}_{n}=\mathscr{H}_{n}(e, \boldsymbol{\kappa})$.

Let $\mathfrak{S}_{n}$ be the symmetric group on $\{1,2, \ldots, n\}$. Then $\mathfrak{S}_{n}$ is a Coxeter group and $\left\{s_{1}, \ldots, s_{n-1}\right\}$ is its standard set of Coxeter generators, where $s_{i}=(i, i+1)$ for $1 \leq i<$ $n$. Let $\ell: \mathfrak{S}_{n} \longrightarrow \mathbb{N}$ be the length function on $\mathfrak{S}_{n}$ so that $\ell(w)=k$ if $k$ is minimal such that $w=s_{i_{1}} \ldots s_{i_{k}}$, for some $s_{i_{j}}$ with $1 \leq i_{j}<n$. If $w=s_{i_{1}} \ldots s_{i_{k}}$, with $k=\ell(w)$, then set $T_{w}=T_{i_{1}} \ldots T_{i_{k}}$. Then $T_{w}$ depends only on $w$, and not on the choice of reduced expression $w=s_{i_{1}} \ldots s_{i_{k}}$, because the braid relations hold in $\mathscr{H}_{n}$; see, for example, [18, Theorem 1.8].

Let $\mathscr{H}_{\xi}\left(\mathfrak{S}_{n}\right)$ be the $R$-submodule of $\mathscr{H}_{n}$ spanned by $\left\{T_{w} \mid w \in \mathfrak{S}_{n}\right\}$. Then $\mathscr{H}_{\xi}\left(\mathfrak{S}_{n}\right)$ is isomorphic to the Iwahori-Hecke algebra of $\mathfrak{S}_{n}$ with parameter $\xi$ by [2, Cor. 3.11].

In order to define the bases of $\mathscr{H}_{n}$ which underpin this paper we now review the combinatorics of multipartitions and tableaux. Recall that a multicomposition of $n$ is an $\ell$-tuple $\boldsymbol{\mu}=\left(\mu^{(1)}, \ldots, \mu^{(\ell)}\right)$ of compositions such that $\left|\mu^{(1)}\right|+\cdots+\left|\mu^{(\ell)}\right|=n$. For each multicomposition let $\mathfrak{S}_{\mu}=\mathfrak{S}_{\mu^{(1)}} \times \cdots \times \mathfrak{S}_{\mu^{(\ell)}}$ be the corresponding parabolic, or Young subgroup, of $\mathfrak{S}_{n}$ where we use the natural embedding $\mathfrak{S}_{\mu} \hookrightarrow \mathfrak{S}_{n}$.

A multipartition of $n$ is a multicomposition $\boldsymbol{\mu}=\left(\mu^{(1)}, \ldots, \mu^{(\ell)}\right)$ of $n$ such that each component $\mu^{(l)}$ is a partition, for $1 \leq l \leq \ell$. The set of multicompositions of $n$ becomes a poset under the dominance order $\unrhd$ where, if $\boldsymbol{\lambda}$ and $\boldsymbol{\mu}$ are multicompositions of $n$, then 
$\lambda \unrhd \mu$ if

$$
\sum_{k=1}^{l-1}\left|\lambda^{(k)}\right|+\sum_{j=1}^{i} \lambda_{j}^{(l)} \geq \sum_{k=1}^{l-1}\left|\mu^{(k)}\right|+\sum_{j=1}^{i} \mu_{j}^{(l)}
$$

for $1 \leq l \leq \ell$ and $i \geq 1$. If $\boldsymbol{\lambda} \unrhd \boldsymbol{\mu}$ and $\boldsymbol{\lambda} \neq \boldsymbol{\mu}$ then we write $\boldsymbol{\lambda} \triangleright \boldsymbol{\mu}$. Let $\mathscr{P}_{n}$ be the poset of the multipartitions of $n$ ordered by dominance.

We identify the multipartition $\boldsymbol{\mu}$ with its diagram

$$
[\boldsymbol{\mu}]=\left\{(r, c, l) \mid 1 \leq c \leq \mu_{r}^{(l)}, r \geq 1 \text { and } 1 \leq l \leq \ell\right\} .
$$

In this way, we will talk of the rows, columns and components of $\boldsymbol{\mu}$. If $\boldsymbol{\mu} \in \mathscr{P}_{n}$ let $\boldsymbol{\mu}^{\prime}=$ $\left(\mu^{(\ell)^{\prime}}, \ldots, \mu^{(1)^{\prime}}\right)$ be the conjugate multipartition which is obtained from $\boldsymbol{\mu}$ by reversing the order of its components and then swapping the rows and columns in each component. We frequently identify $\boldsymbol{\mu}$ and its diagram $[\boldsymbol{\mu}]$, which we think of as an $\ell$-tuple of arrays of boxes in the plane.

Let $\boldsymbol{\mu}$ be a multicomposition of $n$. A $\boldsymbol{\mu}$-tableau is a map $\mathfrak{t}:[\boldsymbol{\mu}] \longrightarrow\{1,2, \ldots, n\}$. We think of $\mathfrak{t}$ as a labelling of the diagram of $\boldsymbol{\mu}$ and we define Shape $(\mathfrak{t})=\boldsymbol{\mu}$. A $\boldsymbol{\mu}$-tableau $\mathfrak{t}$ is row standard if $\mathfrak{t}(r, c, l)<\mathfrak{t}(r, c+1, l)$ whenever $(r, c, l),(r, c+1, l) \in[\boldsymbol{\mu}]$. Let $\operatorname{RStd}(\boldsymbol{\mu})$ be the set of row standard $\boldsymbol{\mu}$-tableau. The conjugate of the $\boldsymbol{\mu}$-tableau $\mathfrak{t}$ is the $\boldsymbol{\mu}^{\prime}$-tableau which is obtained from $\mathfrak{t}$ by reversing its components and then swapping its rows and columns in each component.

Suppose now that $\boldsymbol{\mu}$ is a multipartition. Then a $\boldsymbol{\mu}$-tableau $\mathfrak{t}$ is standard if $\mathfrak{t}$ and $\mathfrak{t}^{\prime}$ are both row standard tableaux. Let $\operatorname{Std}(\boldsymbol{\mu})$ be the set of standard $\boldsymbol{\mu}$-tableaux and $\operatorname{Std}^{2}(\boldsymbol{\mu})=$ $\{(\mathfrak{s}, \mathfrak{t}) \mid \mathfrak{s}, \mathfrak{t} \in \operatorname{Std}(\boldsymbol{\mu})\}$ be the set of pairs of standard $\boldsymbol{\mu}$-tableaux. For convenience we set

$$
\operatorname{Std}\left(\mathscr{P}_{n}\right)=\bigcup_{\boldsymbol{\mu} \in \mathscr{P}_{n}} \operatorname{Std}(\boldsymbol{\mu}) \text { and } \operatorname{Std}^{2}\left(\mathscr{P}_{n}\right)=\bigcup_{\boldsymbol{\mu} \in \mathscr{P}_{n}} \operatorname{Std}^{2}(\boldsymbol{\mu})
$$

Suppose that $\mathfrak{s}$ is a row standard $\boldsymbol{\lambda}$-tableau and that $\mathfrak{t}$ is a row standard $\boldsymbol{\mu}$-tableau, for multicompositions $\boldsymbol{\lambda}$ and $\boldsymbol{\mu}$ of $n$. For each non-negative integer $m$ define $\mathfrak{s}_{m}$ and $\mathfrak{t}_{m}$ to be the subtableaux of $\mathfrak{s}$ and $\mathfrak{t}$, respectively, which contain $\{1,2, \ldots, m\}$. Then $\mathfrak{s}$ dominates $\mathfrak{t}$, and we write $\mathfrak{s} \unrhd \mathfrak{t}$, if

$$
\operatorname{Shape}\left(\mathfrak{s}_{m}\right) \unrhd \operatorname{Shape}\left(\mathfrak{t}_{m}\right), \quad \text { for } 1 \leq m \leq n .
$$

It is straightforward to check that $\mathfrak{s} \unrhd \mathfrak{t}$ if and only if $\mathfrak{t}^{\prime} \unrhd \mathfrak{s}^{\prime}$. Observe also that $\boldsymbol{\lambda} \unrhd \boldsymbol{\mu}$ if and only if $\mathfrak{t}^{\boldsymbol{\lambda}} \unrhd \mathfrak{t}^{\boldsymbol{\mu}}$.

We extend the dominance order to $\operatorname{Std}^{2}\left(\mathscr{P}_{n}\right)$ in two ways by declaring that if $(\mathfrak{s}, \mathfrak{t}) \in$ $\operatorname{Std}^{2}(\boldsymbol{\lambda})$ and $(\mathfrak{u}, \mathfrak{v}) \in \operatorname{Std}^{2}(\boldsymbol{\mu})$ then

$$
\begin{array}{ll}
(\mathfrak{s}, \mathfrak{t}) \unrhd(\mathfrak{u}, \mathfrak{v}) & \text { if } \boldsymbol{\lambda} \triangleright \boldsymbol{\mu} \text { or } \boldsymbol{\lambda}=\boldsymbol{\mu} \text { and } \mathfrak{s} \unrhd \mathfrak{u} \text { and } \mathfrak{t} \unrhd \mathfrak{v}, \\
(\mathfrak{s}, \mathfrak{t}) \unrhd(\mathfrak{u}, \mathfrak{v}) \quad \text { if } \mathfrak{s} \unrhd \mathfrak{u} \text { and } \mathfrak{t} \unrhd \mathfrak{v} .
\end{array}
$$

By definition, $(\mathfrak{s}, \mathfrak{t}) \geq(\mathfrak{u}, \mathfrak{v})$ implies that $(\mathfrak{s}, \mathfrak{t}) \unrhd(\mathfrak{u}, \mathfrak{v})$, but the converse is false in general. As above, we write $(\mathfrak{s}, \mathfrak{t}) \triangleright(\mathfrak{u}, \mathfrak{v})$, and $(\mathfrak{s}, \mathfrak{t}) \triangleright(\mathfrak{u}, \mathfrak{v})$, if $(\mathfrak{s}, \mathfrak{t}) \neq(\mathfrak{u}, \mathfrak{v})$ and $(\mathfrak{s}, \mathfrak{t}) \unrhd(\mathfrak{u}, \mathfrak{v})$ and $(\mathfrak{s}, \mathfrak{t}) \unrhd(\mathfrak{u}, \mathfrak{v})$, respectively. The partial order $\unrhd$ is the strong dominance order on $\operatorname{Std}^{2}\left(\mathscr{P}_{n}\right)$.

Suppose that $\boldsymbol{\mu}$ is a multicomposition of $n$ and define $\mathfrak{t}^{\mu}$ to be the unique row standard $\boldsymbol{\mu}$-tableau such that $\mathfrak{t}^{\boldsymbol{\mu}} \unrhd \mathfrak{s}$ whenever $\mathfrak{s}$ is a row standard $\boldsymbol{\mu}$-tableau. That is, $\mathfrak{t}^{\boldsymbol{\mu}}$ is the $\boldsymbol{\mu}$-tableau with the numbers $1,2, \ldots, n$ entered in order from left to right along the rows of each component of $\mathfrak{t}^{\boldsymbol{\mu}}$. Next if $\mathfrak{s} \in \operatorname{Std}(\boldsymbol{\mu})$ let $d(\mathfrak{s})$ be the unique permutation in $\mathfrak{S}_{n}$ such that $\mathfrak{s}=\mathfrak{t}^{\boldsymbol{\mu}} d(\mathfrak{s})$. 
We are now ready to define the first bases of $\mathscr{H}_{n}$ that we will need. Let $*: \mathscr{H}_{n} \longrightarrow \mathscr{H}_{n}$ be the unique anti-isomorphism of $\mathscr{H}_{n}$ which fixes each of the generators in Definition 2.1

Let $\boldsymbol{\mu}$ be a multicomposition. Following [10,20], set

$$
\begin{array}{ll}
u_{\boldsymbol{\mu}}^{+}=\prod_{k=2}^{l} \prod_{m=1}^{\left|\mu^{(1)}\right|+\cdots+\left|\mu^{(k-1)}\right|}\left(L_{m}-Q_{k}\right), & x_{\boldsymbol{\mu}}=\sum_{w \in \mathfrak{S}_{\mu}} T_{w}, \\
u_{\boldsymbol{\mu}}^{-}=\prod_{k=1}^{\ell-1} \prod_{m=1}^{\left|\mu^{(1)}\right|+\cdots+\left|\mu^{(\ell-k+1)}\right|}\left(L_{m}-Q_{k}\right), \quad y_{\boldsymbol{\mu}}=\sum_{w \in \mathfrak{S}_{\mu}}(-\xi)^{-\ell(w)} T_{w},
\end{array}
$$

and let $m_{\boldsymbol{\mu}}=u_{\boldsymbol{\mu}}^{+} x_{\boldsymbol{\mu}}$ and $n_{\boldsymbol{\mu}}=u_{\boldsymbol{\mu}}^{-} y_{\boldsymbol{\mu}}$. Finally, if $\boldsymbol{\mu}$ is a multipartition define

$$
m_{\mathfrak{s t}}=T_{d(\mathfrak{s})}^{*} m_{\boldsymbol{\mu}} T_{d(\mathfrak{t})} \quad \text { and } \quad n_{\mathfrak{s t}}=T_{d(\mathfrak{s})}^{*} n_{\boldsymbol{\mu}} T_{d(\mathfrak{t})},
$$

for $(\mathfrak{s}, \mathfrak{t}) \in \operatorname{Std}^{2}\left(\mathscr{P}_{n}\right)$. It follows easily from the relations in $\mathscr{H}_{n}$ (see [10 Remark 3.7]), that the elements $x_{\boldsymbol{\mu}}$ and $u_{\boldsymbol{\mu}}^{+}$, and $y_{\boldsymbol{\mu}}$ and $u_{\boldsymbol{\mu}}^{-}$, commute, so that $m_{\mathfrak{s t}}^{*}=m_{\mathfrak{t s}}$ and $n_{\mathfrak{s t}}^{*}=n_{\mathfrak{t s} \text {. }}$. We have the following important and well-known result.

\subsection{Theorem. Suppose that $R$ is an integral domain. Then:}

a) $\left\{m_{\mathfrak{s t}} \mid(\mathfrak{s}, \mathfrak{t}) \in \operatorname{Std}^{2}\left(\mathscr{P}_{n}\right)\right\}$ is a cellular basis of $\mathscr{H}_{n}$.

b) $\left\{n_{\mathfrak{s t}} \mid(\mathfrak{s}, \mathfrak{t}) \in \operatorname{Std}^{2}\left(\mathscr{P}_{n}\right)\right\}$ is a cellular basis of $\mathscr{H}_{n}$.

Part (a) is proved in [10, Theorem 3.26] and [3. Theorem 6.3] for the non-degenerate $(\xi \neq 1)$ and degenerate $(\xi=1)$ cases, respectively. Part (b) is can be proved in the same way or arguing by specialization from the case where $\mathscr{H}_{n}$ is defined over a 'generic' ground ring in which case $\mathscr{H}_{n}$ has a $\mathbb{Z}$-linear automorphism which interchanges these two bases; see, for example, [20. (3.1)].

2.2. Seminormal forms and strong dominance. In this subsection we give a necessary condition for the product $m_{\mathfrak{s t}} n_{\mathfrak{u} \mathfrak{v}}$ to be non-zero. To prove this we show that the transition matrices between the standard and seminormal bases of $\mathscr{H}_{n}$ are ordered by strong dominance, a theme that continues throughout this paper.

Suppose that $(\mathfrak{s}, \mathfrak{t}) \in \operatorname{Std}^{2}\left(\mathscr{P}_{n}\right)$ and $1 \leq k \leq n$. If $k$ appears in row $r$ and column $c$ of $\mathfrak{t}^{(l)}$ then define

$$
\operatorname{cont}_{\mathrm{t}}(k)= \begin{cases}\xi^{c-r} Q_{l}, & \text { if } \xi \neq 1, \\ c-r+Q_{l}, & \text { if } \xi=1 .\end{cases}
$$

Then by [14, Prop. 3.7] and [3. Lemma 6.6], corresponding to the cases $\xi \neq 1$ and $\xi=1$, respectively,

$$
m_{\mathfrak{s t}} L_{k}=\operatorname{cont}_{\mathfrak{t}}(k) m_{\mathfrak{s t}}+\sum_{\substack{(\mathfrak{u}, \mathfrak{v}) \in \operatorname{Std}^{2}\left(\mathscr{P}_{n}\right) \\(\mathfrak{u}, \mathfrak{v}) \triangleright(\mathfrak{s}, \mathfrak{t})}} r_{\mathfrak{u v}} m_{\mathfrak{u v}}
$$

for some $r_{\mathfrak{u v}} \in R$.

For the rest of this subsection we assume that $R=\mathcal{K}$ is a field and that $\mathscr{H}_{n}=\mathscr{H}_{n}^{\mathcal{K}}$ is semisimple. Equivalently, we assume that if $\mathfrak{s}, \mathfrak{t} \in \operatorname{Std}\left(\mathscr{P}_{n}\right)$ then $\operatorname{cont}_{\mathfrak{s}}(k)=\operatorname{cont}_{\mathfrak{t}}(k)$, for all $1 \leq k \leq n$, if and only if $\mathfrak{s}=\mathfrak{t}$.

The following definition has its origins in the work of Murphy [23]. 
2.4. Definition ( [21, Defn 3.1]). Suppose that $\boldsymbol{\lambda} \in \mathscr{P}_{n}$ and $(\mathfrak{s}, \mathfrak{t}) \in \operatorname{Std}^{2}(\boldsymbol{\lambda})$. Define

$$
F_{\mathfrak{t}}=\prod_{k=1}^{n} \prod_{\substack{\mathfrak{s} \in \operatorname{Std}\left(\mathscr{P}_{n}\right) \\ \operatorname{cont}_{\mathfrak{s}}(k) \neq \operatorname{cont}_{\mathfrak{t}}(k)}} \frac{L_{k}-\operatorname{cont}_{\mathfrak{s}}(k)}{\operatorname{cont}_{\mathfrak{t}}(k)-\operatorname{cont}_{\mathfrak{s}}(k)} \in \mathscr{H}_{n}^{\mathcal{K}} .
$$

Set $f_{\mathfrak{s t}}=F_{\mathfrak{s}} m_{\mathfrak{s t}} F_{\mathfrak{t}}$.

By (2.3), $f_{\mathfrak{s t}}=m_{\mathfrak{s t}}+\sum_{(\mathfrak{u}, \mathfrak{v}) \triangleright(\mathfrak{s}, \mathfrak{t})} r_{\mathfrak{u v}} m_{\mathfrak{u v}}$, for some $r_{\mathfrak{u v}} \in \mathcal{K}$. In particular, applying Theorem 2.2 shows that $\left\{f_{\mathfrak{s t}}\right\}$ is a basis of $\mathscr{H}_{n}^{\mathcal{K}}$. Moreover, if $(\mathfrak{s}, \mathfrak{t}) \in \operatorname{Std}^{2}\left(\mathscr{P}_{n}\right)$ and $1 \leq k \leq n$ then

$$
f_{\mathfrak{u v}} L_{k}=\operatorname{cont}_{\mathfrak{v}}(k) f_{\mathfrak{u v}}
$$

by [20, Prop. 2.6(iii)] and [3, Page 109].

If $\mathfrak{s}$ is a tableau and $1 \leq k \leq n$ define $\operatorname{comp}_{\mathfrak{s}}(k)=c$ if $k$ appears in $\mathfrak{s}^{(c)}$. If $\mathfrak{s}$ and $\mathfrak{t}$ are tableau we write $\operatorname{comp}(\mathfrak{s}) \leq \operatorname{comp}(\mathfrak{t})$ if $\operatorname{comp}_{\mathfrak{s}}(k) \leq \operatorname{comp}_{\mathfrak{t}}(k)$ for $1 \leq k \leq n$. Then $\operatorname{comp}(\mathfrak{s}) \leq \operatorname{comp}(\mathfrak{t})$ whenever $\mathfrak{s} \unrhd \mathfrak{t}$, but the converse is false in general.

The following result is well-known. We include a proof because we do not know of a reference for it.

2.6. Lemma. Suppose that $\boldsymbol{\lambda}, \boldsymbol{\mu} \in \mathscr{P}_{n}$ and $\mathfrak{s} \in \operatorname{Std}(\boldsymbol{\lambda})$ and that there exist two integers $a<b$ which are in the same row of $\mathfrak{t}^{\mu}$ and in the same column of $\mathfrak{s}$. Then there exists an element $w \in \mathfrak{S}_{\{a, a+1, \cdots, b\}}$ and an integer $a \leq c<b$ such that

a) $\mathfrak{s} w$ is standard; and

b) $\ell(d(\mathfrak{s}) w)=\ell(d(\mathfrak{s}))+\ell(w)$; and

c) $c, c+1$ are in the same row of $\mathfrak{t}^{\mu}$ and the same column of $\mathfrak{s} w$.

Proof. By assumption, the integers $a, a+1, \cdots, b$ are all in the same row of $\mathfrak{t}^{\mu}$ so because $\mathfrak{s}$ is standard we may assume, without loss of generality, that $a$ appears in row $r$ of $\mathfrak{s}^{(l)}$ and that $b$ appears in row $r+1$ of $\mathfrak{s}^{(l)}$, where $1 \leq l \leq \ell$. We now argue by induction on $b-a$.

If $b-a=1$ then there is nothing to prove, so suppose that $b-a>1$. Let $c<b$ be maximal such that

a) $\operatorname{comp}_{\mathfrak{s}}(c)<l$ or $\operatorname{comp}_{\mathfrak{s}}(c)=l$ and $c$ appears in the first $r$ rows of $\mathfrak{s}^{(l)}$, and

b) $\operatorname{comp}_{s}(c+1)>l$ or $\operatorname{comp}_{\mathfrak{s}}(c)=l$ and $c+1$ appears below row $r$ of $\mathfrak{s}^{(l)}$.

In particular, this means that the numbers $c+1, c+2, \cdots, b$ all appear 'below' row $r$ of $\mathfrak{s}^{(l)}$. Let $\mathfrak{t}=\mathfrak{s} w$, where $w=(c, c+1, \ldots, b)=s_{b-1} \ldots s_{c} \in \mathfrak{S}_{n}$. Then $\mathfrak{s} \unrhd \mathfrak{t}$ and $\ell(d(\mathfrak{t}))=\ell(d(\mathfrak{s}))+\ell(w)$. The integers $a<c$ are in the same row of $\mathfrak{t}^{\mu}$ and in the same column of $\mathfrak{t}=\mathfrak{s} w$. Note that $c-a<b-a$. The Lemma now follows by induction.

2.7. Proposition. Suppose that $R=\mathcal{K}$ and $\boldsymbol{\lambda}$ is a multicomposition of $n>0$. Then there exist scalars $a_{\mathfrak{u v}}^{\lambda} \in \mathcal{K}$ such that

$$
m_{\boldsymbol{\lambda}}=\sum_{(\mathfrak{u}, \mathfrak{v}) \in \operatorname{Std}^{2}\left(\mathscr{P}_{n}\right)} a_{\mathfrak{u v}}^{\boldsymbol{\lambda}} f_{\mathfrak{u v}}
$$

and $a_{\mathfrak{s t}}^{\boldsymbol{\lambda}} \neq 0$ only if $\operatorname{comp}\left(\mathfrak{t}^{\boldsymbol{\lambda}}\right) \geq \operatorname{comp}(\mathfrak{u}), \operatorname{comp}\left(\mathfrak{t}^{\boldsymbol{\lambda}}\right) \geq \operatorname{comp}(\mathfrak{v})$ and $i$ and $j$ are in different columns of $\mathfrak{u}$ and $\mathfrak{v}$ whenever they are in the same column of $\mathfrak{t}^{\lambda}$.

Proof. We consider only the case when $\xi \neq 1$. The case $\xi=1$ is similar and may be proved using the results of $[3, \S 6]$. The only real difference between the cases $\xi \neq 1$ and $\xi=1$ is the choice of content function: if $\xi \neq 1$ then $\operatorname{cont}_{\mathfrak{v}}(k)=\xi^{c-r} Q_{l}$, when $\mathfrak{v}(r, c, l)=k$, and if $\xi=1$ then, instead, $\operatorname{cont}_{\mathfrak{v}}(k)=c-r+Q_{l}$. Analogous minor 'logarithmic' adjustments are required in the argument below when $\xi=1$. 
By (2.3) there exist $a_{\mathfrak{u v}}^{\lambda} \in \mathcal{K}$ such that we can write

$$
m_{\boldsymbol{\lambda}}=f_{\mathfrak{t}^{\boldsymbol{\lambda}} \mathfrak{t}^{\boldsymbol{\lambda}}}+\sum_{(\mathfrak{u}, \mathfrak{v}) \unrhd\left(\mathfrak{t}^{\boldsymbol{\lambda}}, \mathfrak{t}^{\boldsymbol{\lambda}}\right)} a_{\mathfrak{u v}^{\boldsymbol{\lambda}}}^{f_{\mathfrak{u v}}}
$$

Suppose that $s_{i}=(i, i+1) \in \mathfrak{S}_{\boldsymbol{\lambda}}$, where $1 \leq i<n$. It is well-known and easy to check that $x_{\boldsymbol{\lambda}} T_{i}=\xi x_{\boldsymbol{\lambda}}$ (see, for example, [18. Lemma 3.2]), so that $m_{\boldsymbol{\lambda}} T_{i}=\xi m_{\boldsymbol{\lambda}}$. To compute the action of $T_{i}$ on the right hand side of the last equation we need to recall how $\mathscr{H}_{n}$ acts on its seminormal basis.

By [20, Prop. 2.7], $f_{\mathfrak{u v}} T_{i}=\xi f_{\mathfrak{u v}}$ if $i$ and $i+1$ are in the same row of $\mathfrak{v}$ and $f_{\mathfrak{u v}} T_{i}=$ $-f_{\mathfrak{u v}}$ if $i$ and $i+1$ are in the same column of $\mathfrak{v}$. Otherwise, $i$ and $i+1$ are in different rows and columns of $\mathfrak{v}$ so we set $\mathfrak{t}=\mathfrak{v}(i, i+1)$ and $c_{\mathfrak{v}}=\operatorname{cont}_{\mathfrak{v}}(i)$ and $c_{\mathfrak{t}}=\operatorname{cont}_{\mathfrak{t}}(i)=$ $\operatorname{cont}_{\mathfrak{v}}(i+1)$. Without loss of generality, $\mathfrak{v} \triangleright \mathfrak{t}$. Hence, by [20, Prop. 2.7] we have

$$
\begin{aligned}
f_{\mathfrak{u v}} T_{i} & =\frac{(\xi-1) c_{\mathfrak{t}}}{\left(c_{\mathfrak{t}}-c_{\mathfrak{v}}\right)} f_{\mathfrak{u} \mathfrak{v}}+f_{\mathfrak{u t}}, \\
f_{\mathfrak{u t}} T_{i} & =\frac{\left(\xi c_{\mathfrak{v}}-c_{\mathfrak{t}}\right)\left(c_{\mathfrak{v}}-\xi c_{\mathfrak{t}}\right)}{\left(c_{\mathfrak{v}}-c_{\mathfrak{t}}\right)^{2}} f_{\mathfrak{u v}}+\frac{(\xi-1) c_{\mathfrak{v}}}{\left(c_{\mathfrak{v}}-c_{\mathfrak{t}}\right)} f_{\mathfrak{u t}} .
\end{aligned}
$$

Therefore, $a_{\mathfrak{u} \mathfrak{v}}^{\boldsymbol{\lambda}}=0$ if $i$ and $i+1$ are in the same column. If $\mathfrak{v}$ and $\mathfrak{t}$ are both standard tableau and $\mathfrak{v} \triangleright \mathfrak{t}$ then

$$
\begin{aligned}
\xi a_{\mathfrak{u} \mathfrak{v}}^{\boldsymbol{\lambda}} & =\frac{(\xi-1) c_{\mathfrak{t}}}{\left(c_{\mathfrak{t}}-c_{\mathfrak{v}}\right)} a_{\mathfrak{u} \mathfrak{v}}^{\boldsymbol{\lambda}}+\frac{\left(\xi c_{\mathfrak{v}}-c_{\mathfrak{t}}\right)\left(c_{\mathfrak{v}}-\xi c_{\mathfrak{t}}\right)}{\left(c_{\mathfrak{v}}-c_{\mathfrak{t}}\right)^{2}} a_{\mathfrak{u} \mathfrak{t}}^{\boldsymbol{\lambda}} \\
\xi a_{\mathfrak{u} \mathfrak{t}}^{\boldsymbol{\lambda}} & =a_{\mathfrak{u} \mathfrak{v}}^{\boldsymbol{\mu}}+\frac{(\xi-1) c_{\mathfrak{v}}}{\left(c_{\mathfrak{v}}-c_{\mathfrak{t}}\right)} a_{\mathfrak{u} \mathfrak{t}}^{\boldsymbol{\lambda}} .
\end{aligned}
$$

Solving these equations shows that $a_{\mathfrak{u v}}^{\boldsymbol{\lambda}}=\left(c_{\mathfrak{v}}-\xi c_{\mathfrak{t}}\right) /\left(c_{\mathfrak{v}}-c_{\mathfrak{t}}\right) \cdot a_{\mathfrak{u t}}^{\boldsymbol{\lambda}}$. In particular, $a_{\mathfrak{u v}}^{\boldsymbol{\lambda}} \neq 0$ if and only if $a_{\mathfrak{u t}}^{\boldsymbol{\lambda}} \neq 0$, since $c_{\mathfrak{v}} \neq c_{\mathfrak{t}}$ and $c_{\mathfrak{v}} \neq \xi c_{\mathfrak{t}}$ (because $\mathscr{H}_{n}^{\mathcal{K}}$ is semisimple), whenever $i$ and $i+1$ are in the same row of $\mathfrak{t}^{\boldsymbol{\lambda}}$ and in different columns of $\mathfrak{v}$. Applying Lemma 2.6 and acting by $\mathfrak{S}_{\boldsymbol{\lambda}}$ now shows that $a_{\mathfrak{u v}}^{\boldsymbol{\lambda}}=0$ whenever there exist $i$ and $j$ which are in the same column of $\mathfrak{v}$ and the same row of $\mathfrak{t}^{\boldsymbol{\lambda}}$.

To complete the proof we need to show that $\operatorname{comp}\left(\mathfrak{t}^{\boldsymbol{\lambda}}\right) \geq \operatorname{comp}(\mathfrak{u})$ and $\operatorname{comp}\left(\mathfrak{t}^{\boldsymbol{\lambda}}\right) \geq$ $\operatorname{comp}(\mathfrak{v})$. In fact, since $m_{\boldsymbol{\lambda}}=m_{\boldsymbol{\lambda}}^{*}$ it is enough to show that $\operatorname{comp}\left(\mathfrak{t}^{\boldsymbol{\lambda}}\right) \geq \operatorname{comp}(\mathfrak{v})$. Let $1 \leq l \leq \ell$ be minimal such that $\left|\lambda^{(l)}\right|>0$. If $l=\ell$ then $\operatorname{comp}\left(\mathfrak{t}^{\boldsymbol{\lambda}}\right) \geq \operatorname{comp}(\mathfrak{v})$ for any tableau $\mathfrak{v}$ so in this case there is nothing to prove. Suppose then that $1 \leq l<\ell$ and fix $(\mathfrak{s}, \mathfrak{t}) \in \operatorname{Std}\left(\mathscr{P}_{n}\right)$ with $a_{\mathfrak{s t}}^{\lambda} \neq 0$. Let $\boldsymbol{\nu}$ be the multicomposition with $\nu^{(\bar{k})}=\lambda^{(k)}$ if $k \neq l, l+1, \nu^{(l)}=(0)$ and where $\nu^{(l+1)}$ is the composition obtained by concatenating $\lambda^{(l)}$ and $\lambda^{(l+1)}$. Let $m=\left|\lambda^{(l)}\right|$. Then $x_{\boldsymbol{\lambda}}=x_{\boldsymbol{\nu}}$ and

$$
m_{\boldsymbol{\lambda}}=m_{\boldsymbol{\nu}} \prod_{k=1}^{m}\left(L_{k}-Q_{l+1}\right)=\sum_{(\mathfrak{u}, \mathfrak{v}) \in \operatorname{Std}^{2}\left(\mathscr{P}_{n}\right)} a_{\mathfrak{u} \mathfrak{v}}^{\nu} f_{\mathfrak{u} \mathfrak{v}} \prod_{k=1}^{m}\left(L_{k}-Q_{l+1}\right) .
$$

Thus, $a_{\mathfrak{u} \mathfrak{v}}^{\boldsymbol{\lambda}}=a_{\mathfrak{u v}}^{\nu}\left(\operatorname{cont}_{\mathfrak{v}}(1)-Q_{l+1}\right) \ldots\left(\operatorname{cont}_{\mathfrak{v}}(m)-Q_{l+1}\right)$ by (2.5). By induction $a_{\mathfrak{u v}}^{\nu} \neq 0$ only if $\operatorname{comp}\left(\mathfrak{t}^{\nu}\right) \geq \operatorname{comp}(\mathfrak{v})$. In particular, if $a_{\mathfrak{u} \mathfrak{v}}^{\nu} \neq 0$ then $\operatorname{comp}_{\mathfrak{v}}(k) \leq \operatorname{comp}_{\mathfrak{t}^{\lambda}}(k)=$ $\operatorname{comp}_{\mathfrak{t}^{\nu}}(k)$ whenever $m<k \leq n$. Consequently, if $\operatorname{comp}\left(\mathfrak{t}^{\boldsymbol{\lambda}}\right) \nsupseteq \operatorname{comp}(\mathfrak{v})$ then there must exist a $k$ with $1 \leq k \leq m$ and $\operatorname{comp}_{\mathfrak{v}}(k)=l+1$. Therefore, since $\mathfrak{v}$ is standard and $\operatorname{comp}_{\mathfrak{v}}\left(k^{\prime}\right) \leq \operatorname{comp}_{\mathfrak{t}^{\nu}}\left(k^{\prime}\right)$, there exists $1 \leq k^{\prime} \leq k$ in the first row and column of $\mathfrak{v}^{(l+1)}$ so that $\operatorname{cont}_{\mathfrak{v}}\left(k^{\prime}\right)=Q_{l+1}$. Consequently, $a_{\mathfrak{u v}}^{\boldsymbol{\lambda}}=0$ and $f_{\mathfrak{u v}}$ does not appear in $m_{\boldsymbol{\lambda}}$ in this case. It follows that $a_{\mathfrak{u v}}^{\boldsymbol{\lambda}} \neq 0$ only if $\operatorname{comp}\left(\mathfrak{t}^{\boldsymbol{\lambda}}\right) \geq \operatorname{comp}(\mathfrak{v})$ as required. 
2.8. Corollary. Suppose that $\boldsymbol{\mu} \in \mathscr{P}_{n}$ is a multipartition of $n$. Then there exist scalars $a_{\mathfrak{u v}} \in \mathcal{K}$ such that

$$
m_{\boldsymbol{\mu}}=\sum_{\substack{(\mathfrak{u}, \mathfrak{v}) \in \operatorname{Std}^{2}\left(\mathscr{P}_{n}\right) \\(\mathfrak{u}, \mathfrak{v})}} a_{\mathfrak{u v}\left(\mathfrak{t}^{\mu}, \mathfrak{t}^{\mu}\right)} f_{\mathfrak{u v}}
$$

Proof. Suppose that $a_{\mathfrak{u v}} \neq 0$ for some $(\mathfrak{u}, \mathfrak{v}) \in \operatorname{Std}^{2}\left(\mathscr{P}_{n}\right)$. By Proposition $2.7 a_{\mathfrak{u v}} \neq 0$ only if $\operatorname{comp}(\mathfrak{v}) \leq \operatorname{comp}\left(\mathfrak{t}^{\mu}\right)$ and $i$ and $j$ are in different columns of $\mathfrak{v}$ whenever they are in the same row of $\mathfrak{t}^{\mu}$. These two conditions imply that if $1 \leq m \leq n$ then the node containing $m$ in $\mathfrak{v}$ is never below the node which contains $m$ in $\mathfrak{t}^{\mu}$. This implies that $\mathfrak{v} \unrhd \mathfrak{t}^{\mu}$. Similarly, or by applying the involution $*, \mathfrak{u} \unrhd \mathfrak{t}^{\mu}$.

We want to generalize result to an arbitrary basis element $m_{\mathfrak{s t}}$. To do this we use the following two technical results, the first of which is due to Murphy [24]. First, recall from [18. Theorem 3.8] that if $\hat{\mathfrak{v}}$ and $\mathfrak{v}$ are two row standard $\nu$-tableaux then $\mathfrak{v} \unrhd \hat{\mathfrak{v}}$ if and only if $d(\mathfrak{v}) \leq d(\hat{\mathfrak{v}})$ where $\leq$ is the Bruhat order on $\mathfrak{S}_{n}$. That is, $u \leq v$ if $u$ has a reduced expression which is a subexpression of some reduced expression of $v$.

2.9. Lemma (Murphy [24, Lemma 3.5]). Suppose that $\hat{\mathfrak{v}} \in \operatorname{RStd}(\boldsymbol{\mu}), \mathfrak{s} \in \operatorname{Std}(\boldsymbol{\mu})$ and $u, w \in \mathfrak{S}_{n}$ such that $u \leq w, \hat{\mathfrak{v}} \unrhd \mathfrak{s}, \hat{\mathfrak{v}} u$ is row standard, $\mathfrak{s} \triangleright \mathfrak{s} w$ and $\ell(d(\mathfrak{s}) w)=\ell(d(\mathfrak{s}))+$ $\ell(w)$. Then $\hat{\mathfrak{v}} u \unrhd \mathfrak{s} w$.

When comparing our statement of Lemma 2.9 with Murphy's result note that Murphy considers row standard $\mu$-tableaux, where $\mu$ is a composition. Let $\mu^{\vee}=\mu^{(1)} \vee \cdots \vee \mu^{(\ell)}$ be the composition obtained by concatenating the parts of $\mu$. Then any row standard $\boldsymbol{\mu}$ tableau $\mathfrak{t}$ corresponds to the row standard $\mu^{\vee}$-tableau $\mathfrak{t}^{\vee}=\mathfrak{t}^{(1)} \vee \cdots \vee \mathfrak{t}^{(\ell)}$ obtained by concatenating the rows of $t$. This observation allows us to rewrite Murphy's lemma in the form above.

2.10. Lemma. Suppose that $\mathfrak{u}, \mathfrak{v} \in \operatorname{Std}(\boldsymbol{\nu})$ and $\mathfrak{t} \in \operatorname{Std}(\boldsymbol{\mu})$ are standard tableaux such that $\mathfrak{v} \unrhd \mathfrak{t}^{\mu}$ and that $x \in \mathfrak{S}_{n}$ with $x \leq d(\mathfrak{t})$. Then $m_{\mathfrak{u v}} T_{x}$ can be written as a linear combination of terms $m_{\mathfrak{c} d}$ such that $\mathfrak{c} \unrhd \mathfrak{u}$ and $\mathfrak{d} \unrhd \mathfrak{t}$.

Proof. Observe that $x_{\boldsymbol{\nu}} \mathscr{H}_{\xi}\left(\mathfrak{S}_{n}\right)$ is the permutation module for $\mathscr{H}_{\xi}\left(\mathfrak{S}_{n}\right)$, in the sense of [18. Chapt. 3], which is indexed by the composition $\nu^{\vee}$ which is obtained by concatenating the components of $\boldsymbol{\nu}$. By [18 Cor. 3.4], $\left\{x_{\boldsymbol{\nu}} T_{d(\mathfrak{b})} \mid \mathfrak{b} \in \operatorname{RStd}(\boldsymbol{\nu})\right\}$ is a basis of the $\mathscr{H}_{\xi}\left(\mathfrak{S}_{n}\right)$-module $x_{\nu} \mathscr{H}_{\xi}\left(\mathfrak{S}_{n}\right)$. Moreover, the same result shows that if $1 \leq i<n$ then

$$
x_{\boldsymbol{\nu}} T_{d(\mathfrak{v})} T_{i}= \begin{cases}\xi x_{\boldsymbol{\nu}} T_{d(\mathfrak{v})}, & \text { if } \mathfrak{v} s_{i} \notin \operatorname{RStd}(\boldsymbol{\nu}), \\ x_{\boldsymbol{\nu}} T_{d(\mathfrak{v}) s_{i}}, & \text { if } \mathfrak{v} \triangleright \mathfrak{v} s_{i} \in \operatorname{RStd}(\boldsymbol{\nu}), \\ \xi x_{\boldsymbol{\nu}} T_{d(\mathfrak{v}) s_{i}}+(\xi-1) x_{\boldsymbol{\nu}} T_{d(\mathfrak{v})}, & \text { if } \mathfrak{v} \triangleleft \mathfrak{v} s_{i} \in \operatorname{RStd}(\boldsymbol{\nu}) .\end{cases}
$$

We note that if $\mathfrak{v} s_{i} \in \operatorname{RStd}(\boldsymbol{\nu})$ then either $\mathfrak{v} \triangleright \mathfrak{v} s_{i}$ or $\mathfrak{v} s_{i} \triangleright \mathfrak{v}$. Applying (2.11) recursively, we see that $x_{\boldsymbol{\nu}} T_{d(\mathfrak{v})} T_{x}$ is a linear combination of terms of the form $x_{\boldsymbol{\nu}} T_{d(\mathfrak{b})}$ where $\mathfrak{b}=$ $\mathfrak{t}^{\nu} d(\hat{\mathfrak{v}}) u \in \operatorname{RStd}(\boldsymbol{\nu}), \hat{\mathfrak{v}}$ is a row standard $\boldsymbol{\nu}$-tableau such that $\hat{\mathfrak{v}} \unrhd \mathfrak{v}, u \leq x \leq d(\mathfrak{t})$ and $\ell(d(\hat{\mathfrak{v}}) u)=\ell(d(\hat{\mathfrak{v}}))+\ell(u)$. Thus, we have $\hat{\mathfrak{v}} \in \operatorname{RStd}(\boldsymbol{\nu}), \mathfrak{b}=\hat{\mathfrak{v}} u \in \operatorname{RStd}(\boldsymbol{\nu})$ and $\hat{\mathfrak{v}} \unrhd \mathfrak{v} \unrhd \mathfrak{t}^{\mu}$. Hence, setting $\mathfrak{s}=\mathfrak{t}^{\mu}$ and $w=d(\mathfrak{t})$ we see that all of the conditions in Murphy's Lemma 2.9 are satisfied so that $\mathfrak{b}=\hat{\mathfrak{v}} u \unrhd \mathfrak{s} w=\mathfrak{t}$. That is, $x_{\boldsymbol{\nu}} T_{d(\mathfrak{v})} T_{x}$ can be written as a linear combination of $x_{\boldsymbol{\nu}} T_{d(\mathfrak{b})}$ where $\mathfrak{b} \in \operatorname{RStd}(\boldsymbol{\nu})$ and $\mathfrak{b} \unrhd \mathfrak{t}$.

We have shown that $m_{\mathfrak{u v}} T_{x}$ can we written as a linear combination of terms of the form $m_{\mathfrak{u} \mathfrak{b}}$, where $\mathfrak{b} \unrhd \mathfrak{t}$ is row standard. Hence, by [10, Prop. 3.18] we can write $m_{\mathfrak{u v}} T_{x}$ as a linear combination of elements of the form $m_{\mathfrak{c d}}$ where $\mathfrak{c} \unrhd \mathfrak{u}$ and $\mathfrak{d} \unrhd \mathfrak{t}$. (Note that the standard tableaux $\mathfrak{c}$ and $\mathfrak{d}$ do not necessarily have shape $\boldsymbol{\mu}$.) This completes the proof. 
2.12. Theorem. Suppose that $R=\mathcal{K}$ and $(\mathfrak{s}, \mathfrak{t}) \in \operatorname{Std}^{2}\left(\mathscr{P}_{n}\right)$. Then:

a) There exist scalars $a_{\mathfrak{u v}} \in \mathcal{K}$ such that

$$
m_{\mathfrak{s t}}=f_{\mathfrak{s t}}+\sum_{\substack{(\mathfrak{u}, \mathfrak{v}) \in \operatorname{Std}^{2}\left(\mathscr{P}_{n}\right) \\(\mathfrak{u}, \mathfrak{v}) \mathbf{v}(\mathfrak{s}, \mathfrak{t})}} a_{\mathfrak{u v}} f_{\mathfrak{u v}} .
$$

b) There exist scalars $b_{\mathfrak{u v}} \in \mathcal{K}$ such that

$$
f_{\mathfrak{s t}}=m_{\mathfrak{s t}}+\sum_{\substack{(\mathfrak{u}, \mathfrak{v}) \in \operatorname{Std}^{2}\left(\mathscr{P}_{n}\right) \\(\mathfrak{u}, \mathfrak{v}) \mathbf{( s , \mathfrak { t } )}}} b_{\mathfrak{u v}} m_{\mathfrak{u v}} .
$$

Proof. We first prove part (b) using induction on $\boldsymbol{\Sigma}$. If $\boldsymbol{\eta}=((n),(0), \ldots,(0))$ then, directly from the definitions, $f_{\mathfrak{t}^{\eta} \mathfrak{t}^{\eta}}=m_{\mathfrak{t}^{\eta} \mathfrak{t}^{\eta}}=m_{\eta}$. Hence, (b) is automatically true in this case. Notice also that $\left(\mathfrak{t}^{\eta}, \mathfrak{t}^{\eta}\right) \geq(\mathfrak{s}, \mathfrak{t})$, for all $(\mathfrak{s}, \mathfrak{t}) \in \operatorname{Std}^{2}\left(\mathscr{P}_{n}\right)$. Suppose now that $(\mathfrak{s}, \mathfrak{t}) \in \operatorname{Std}^{2}(\boldsymbol{\mu})$ and $(\mathfrak{s}, \mathfrak{t}) \neq\left(\mathfrak{t}^{\eta}, \mathfrak{t}^{\eta}\right)$. Then, by Corollary 2.8 and induction, there exist scalars $a_{\mathfrak{u v}} \in \mathcal{K}$ such that

$$
f_{\mathfrak{t} \mu_{\mathfrak{t}} \mu}=m_{\mu}+\sum_{(\mathfrak{u}, \mathfrak{v}) \backslash\left(\mathfrak{t}^{\mu}, \mathfrak{t}^{\mu}\right)} a_{\mathfrak{u v}} m_{\mathfrak{u v}} .
$$

Suppose that $(\mathfrak{s}, \mathfrak{t}) \in \operatorname{Std}^{2}(\boldsymbol{\mu})$. By [20, Proposition 4.1 and Lemma 4.3], there exists elements $\Phi_{\mathfrak{s}}, \Phi_{\mathfrak{t}} \in \mathscr{H}_{\xi}\left(\mathfrak{S}_{n}\right)$ such that $f_{\mathfrak{s t}}=\Phi_{\mathfrak{s}}^{*} f_{\mathfrak{t}^{\mu} \mathfrak{t}} \Phi_{\mathfrak{t}}$. (In [20] this is proved only in the case when $\xi \neq 1$. The case when $\xi=1$ follows by exactly the same argument.) Therefore, by the last displayed equation,

$$
f_{\mathfrak{s t}}=\Phi_{\mathfrak{s}}^{*} f_{\mathfrak{t} \mu \mathfrak{t} \mu} \Phi_{\mathfrak{t}}=\sum_{(\mathfrak{u}, \mathfrak{v})} \sum_{\left(\mathfrak{t}^{\mu}, \mathfrak{t}^{\mu}\right)} a_{\mathfrak{u v}} \Phi_{\mathfrak{s}}^{*} m_{\mathfrak{u v}} \Phi_{\mathfrak{t}}
$$

where for convenience we set $a_{\mathfrak{t}^{\mu} \mathfrak{t}^{\mu}}=1$. By the argument of [20, Proposition 4.1(ii)], $\Phi_{\mathfrak{t}}=\sum_{b \leq d(\mathfrak{t})} p_{\mathfrak{t} b} T_{b}$, for some $p_{\mathfrak{t} b} \in \mathcal{K}$ where, in the sum, $b \in \mathfrak{S}_{n}$ (with $p_{\mathfrak{t} d(\mathfrak{t})}=1$ ). By Lemma 2.10 we can write $m_{\mathfrak{u v}} T_{b}$ as a linear combination of elements of the form $m_{\mathfrak{c} d}$ with $\mathfrak{c} \unrhd \mathfrak{u}$ and $\mathfrak{d} \unrhd \mathfrak{t}$. Hence, we can write $m_{\mathfrak{u v}} \Phi_{\mathfrak{t}}$ as a linear combination of terms $m_{\mathfrak{c} d}$ with $(\mathfrak{c}, \mathfrak{d}) \geq\left(\mathfrak{t}^{\mu}, \mathfrak{t}\right)$. Applying the left handed version of Lemma 2.10 to each of the terms $\Phi_{\mathfrak{s}}^{*} m_{\mathfrak{c} \mathfrak{d}}$, we see that each $\Phi_{\mathfrak{s}}^{*} m_{\mathfrak{u v}} \Phi_{\mathfrak{t}}$ can be written as a linear combination of elements of the form $m_{\mathfrak{a} \mathfrak{b}}$ with $(\mathfrak{a}, \mathfrak{b}) \geq(\mathfrak{s}, \mathfrak{t})$. Hence, $f_{\mathfrak{s t}}$ can be written as a linear combination of elements $m_{\mathfrak{a} \mathfrak{b}}$ with $(\mathfrak{a}, \mathfrak{b}) \geq(\mathfrak{s}, \mathfrak{t})$ giving (b). Inverting the equations in (b) gives (a), completing the proof.

We can now prove the promised criterion for the product $m_{\mathfrak{s t}} n_{\mathfrak{u v}}$ to be non-zero. Notice that unlike Theorem 2.12, which requires $R=\mathcal{K}$, the next results are valid over an arbitrary integral domain.

2.13. Corollary. Suppose that $R$ is an integral domain and that $(\mathfrak{s}, \mathfrak{t}),(\mathfrak{u}, \mathfrak{v}) \in \operatorname{Std}^{2}\left(\mathscr{P}_{n}\right)$. Then:

a) $m_{\mathfrak{s t}} n_{\mathfrak{u v}} \neq 0$ only if $\mathfrak{u}^{\prime} \unrhd \mathfrak{t}$, and,

b) $n_{\mathfrak{u v}} m_{\mathfrak{s t}} \neq 0$ only if $\mathfrak{v}^{\prime} \unrhd \mathfrak{s}$.

Proof. Parts (a) and (b) are equivalent by applying the anti-isomorphism $*$ of $\mathscr{H}_{n}$ which fixes each generator, so we prove only (a). Let $K$ be the field of fractions of $R$. Then by embedding $\mathscr{H}_{n}^{R}$ into $\mathscr{H}_{n}^{K}$ and choosing a suitable modular system $(\mathcal{O}, \mathcal{K}, K)$ (see [13, §4.2] for example), we can reduce to the case where $R=\mathcal{K}$. Following [20], if $(\mathfrak{u}, \mathfrak{v}) \in \operatorname{Std}^{2}\left(\mathscr{P}_{n}\right)$ then define $g_{\mathfrak{u v}}=F_{\mathfrak{u}^{\prime}} n_{\mathfrak{u v}} F_{\mathfrak{v}^{\prime}}$. Then by repeating the arguments of 
Theorem 2.12 (or by applying a suitable automorphism in the generic case as in the proof of [20. Prop. 3.4]), it follows that

$$
n_{\mathfrak{u v}}=g_{\mathfrak{u v}}+\sum_{(\mathfrak{c}, \mathfrak{d}) \backslash(\mathfrak{u}, \mathfrak{v})} d_{\mathfrak{c o}} g_{\mathfrak{c} \mathfrak{d}},
$$

for some scalars $d_{\mathfrak{c} \mathfrak{d}} \in \mathcal{K}$. Hence, by Theorem 2.12 we have

$$
0 \neq m_{\mathfrak{s t}} n_{\mathfrak{u v}}=\left(f_{\mathfrak{s t}}+\sum_{(\mathfrak{a}, \mathfrak{b}) \triangleright(\mathfrak{s}, \mathfrak{t})} c_{\mathfrak{a} \mathfrak{b}} f_{\mathfrak{a} \mathfrak{b}}\right)\left(g_{\mathfrak{u v}}+\sum_{(\mathfrak{c}, \mathfrak{d}) \triangleright(\mathfrak{u}, \mathfrak{v})} d_{\mathfrak{c o}} g_{\mathfrak{c o}}\right) .
$$

Hence, there exist tableaux $(\mathfrak{a}, \mathfrak{b}) \geq(\mathfrak{s}, \mathfrak{t})$ and $(\mathfrak{c}, \mathfrak{d}) \geq(\mathfrak{u}, \mathfrak{v})$ such that $f_{\mathfrak{a} \mathfrak{b}} g_{\mathfrak{c} \mathfrak{d}} \neq 0$. By $[\mathbf{2 0}$, Corollary 3.8], $f_{\mathfrak{a} \mathfrak{b}} g_{\mathfrak{c} \mathfrak{d}} \neq 0$ only if $\mathfrak{c}^{\prime}=\mathfrak{b}$. Therefore, $\mathfrak{u}^{\prime} \unrhd \mathfrak{c}^{\prime}=\mathfrak{b} \unrhd \mathfrak{t}$, so that $\mathfrak{u}^{\prime} \unrhd \mathfrak{t}$ as required.

2.14. Remark. Both the statement and proof of part (b) of Corollary 2.13 is essentially the same as [19. Lemma 5.4]. Unfortunately, in [19] the second author confused the two partial orders $\unrhd$ and $\geq$ on $\operatorname{Std}^{2}\left(\mathscr{P}_{n}\right)$, so all that was actually proved in that paper was that Shape $\left(\mathfrak{u}^{\prime}\right) \unrhd$ Shape $(\mathfrak{t})$ whenever $m_{\mathfrak{s t}} n_{\mathfrak{u} \mathfrak{v}} \neq 0$. As a consequence, the current paper completes the proof of [19. Lemma 5.8] which requires the full strength of Corollary 2.13 (and Lemma2.6).

2.15. Corollary. Suppose that $R$ is an integral domain, $1 \leq k \leq n$ and $(\mathfrak{s}, \mathfrak{t}) \in \operatorname{Std}^{2}\left(\mathscr{P}_{n}\right)$. Then there exist scalars $c_{\mathfrak{u v}} \in R$ such that

$$
m_{\mathfrak{s t}} L_{k}=\operatorname{cont}_{\mathfrak{t}}(k) m_{\mathfrak{s t}}+\sum_{\substack{(\mathfrak{u}, \mathfrak{v}) \in \operatorname{Std}^{2}\left(\mathscr{P}_{n}\right) \\(\mathfrak{u}, \mathfrak{v}) \backslash(\mathfrak{s}, \mathfrak{t})}} c_{\mathfrak{u v}} m_{\mathfrak{u v}} .
$$

Proof. As in the proof of Corollary 2.13 it is enough to consider the case when $R=\mathcal{K}$ and $\mathscr{H}_{n}^{\mathcal{K}}$ is semisimple. Using parts (a) and (b) of Theorem 2.12, to switch between the standard and seminormal bases, together with $(2.5)$ for the second equality we see that

$$
\begin{aligned}
m_{\mathfrak{s t}} L_{k} & =\left(f_{\mathfrak{s t}}+\sum_{\substack{(\mathfrak{u}, \mathfrak{v}) \in \operatorname{Std}^{2}\left(\mathscr{P}_{n}\right) \\
(\mathfrak{u}, \mathfrak{v}) \triangleright(\mathfrak{s}, \mathfrak{t})}} a_{\mathfrak{u v}} f_{\mathfrak{u v}}\right) L_{k} \\
& =\operatorname{cont}_{\mathfrak{t}}(k) f_{\mathfrak{s t}}+\sum_{\substack{(\mathfrak{u}, \mathfrak{v}) \in \operatorname{Std}^{2}\left(\mathscr{P}_{n}\right) \\
(\mathfrak{u}, \mathfrak{v})>(\mathfrak{s}, \mathfrak{t})}} \operatorname{cont}_{\mathfrak{v}}(k) a_{\mathfrak{u v}} f_{\mathfrak{u v}} \\
& =\operatorname{cont}_{\mathfrak{t}}(k) m_{\mathfrak{s t}}+\sum_{\substack{(\mathfrak{u}, \mathfrak{v}) \in \operatorname{Std}^{2}\left(\mathscr{P}_{n}\right) \\
(\mathfrak{u}, \mathfrak{v}) \boldsymbol{( s , \mathfrak { t } )}}} \operatorname{cont}_{\mathfrak{v}}(k) c_{\mathfrak{u v}} m_{\mathfrak{u v}},
\end{aligned}
$$

for some $c_{\mathfrak{u v}} \in \mathcal{K}$. This completes the proof.

We remark that it is not hard to see that Corollary 2.8, Corollary 2.15 and the two statements in Theorem 2.12 are all, in fact, equivalent. There are analogous (equivalent) statements for the basis $\left\{n_{\mathfrak{s t}}\right\}$. We leave the details to the interested reader.

Similarly, one can show that $n_{\mathfrak{s t}} L_{k}=\operatorname{cont}_{\mathfrak{t}^{\prime}}(k) n_{\mathfrak{s t}}$ plus a linear combination of terms $n_{\mathfrak{u} \mathfrak{v}}$ with $(\mathfrak{u}, \mathfrak{v}) \triangleright(\mathfrak{s}, \mathfrak{t})$. 


\section{Cyclotomic Quiver Hecke algebras and graded Specht modules}

We now switch to the cyclotomic quiver Hecke algebras, which were introduced in a series of papers by Khovanov-Lauda [16], Rouquier [25] and Brundan and Kleshchev [6]. These algebras are certain naturally graded algebras which depend on $e$. As we recall, when they are defined over suitable fields, they are isomorphic to the cyclotomic Hecke algebras of the last section if we take $e$ to be equal to the quantum characteristic of the parameter $\xi$ and choose appropriate parameters.

3.1. Cyclotomic Quiver Hecke algebras and graded induction. Recall from the introduction that we have fixed an integer $e \in\{0,2,3,4, \ldots\}$ and that $I=\mathbb{Z} / e \mathbb{Z}$. Let $\Gamma$ be the oriented quiver with vertex set $I$ and directed edges $i \longrightarrow i+1$, for $i \in I$. To the quiver $\Gamma$ we attach the standard Lie theoretic data of a Cartan matrix $\left(a_{i j}\right)_{i, j \in I}$, fundamental weights $\left\{\Lambda_{i} \mid i \in I\right\}$, positive weights $X^{+}=\sum_{i \in I} \mathbb{N} \Lambda_{i}$, positive roots $Q_{+}=\bigoplus_{i \in I} \mathbb{N} \alpha_{i}$ and we let $(\cdot, \cdot)$ be the bilinear form determined by

$$
\left(\alpha_{i}, \alpha_{j}\right)=a_{i j} \quad \text { and } \quad\left(\Lambda_{i}, \alpha_{j}\right)=\delta_{i j}, \quad \text { for } i, j \in I .
$$

More details can be found, for example, in [15, Chapt. 1].

3.1. Definition. The cyclotomic quiver Hecke algebra, or cyclotomic Khovanov-LaudaRouquier algebra, $\mathscr{R}_{n}^{\Lambda}$ of weight $\Lambda$ and type $\Gamma_{e}$ is the unital associative R-algebra with generators

$$
\left\{\psi_{1}, \ldots, \psi_{n-1}\right\} \cup\left\{y_{1}, \ldots, y_{n}\right\} \cup\left\{e(\mathbf{i}) \mid \mathbf{i} \in I^{n}\right\}
$$

and relations

$$
\begin{aligned}
& y_{1}^{\left(\Lambda, \alpha_{i_{1}}\right)} e(\mathbf{i})=0, \quad e(\mathbf{i}) e(\mathbf{j})=\delta_{\mathbf{i j}} e(\mathbf{i}), \quad \sum_{\mathbf{i} \in I^{n}} e(\mathbf{i})=1, \\
& y_{r} e(\mathbf{i})=e(\mathbf{i}) y_{r}, \quad \psi_{r} e(\mathbf{i})=e\left(s_{r} \cdot \mathbf{i}\right) \psi_{r}, \quad y_{r} y_{s}=y_{s} y_{r}, \\
& \psi_{r} y_{s}=y_{s} \psi_{r}, \quad \text { if } s \neq r, r+1, \\
& \psi_{r} \psi_{s}=\psi_{s} \psi_{r}, \quad \text { if }|r-s|>1, \\
& \psi_{r} y_{r+1} e(\mathbf{i})= \begin{cases}\left(y_{r} \psi_{r}+1\right) e(\mathbf{i}), & \text { if } i_{r}=i_{r+1}, \\
y_{r} \psi_{r} e(\mathbf{i}), & \text { if } i_{r} \neq i_{r+1}\end{cases} \\
& y_{r+1} \psi_{r} e(\mathbf{i})= \begin{cases}\left(\psi_{r} y_{r}+1\right) e(\mathbf{i}), & \text { if } i_{r}=i_{r+1}, \\
\psi_{r} y_{r} e(\mathbf{i}), & \text { if } i_{r} \neq i_{r+1}\end{cases} \\
& \psi_{r}^{2} e(\mathbf{i})= \begin{cases}0, & \text { if } i_{r}=i_{r+1}, \\
e(\mathbf{i}), & \text { if } i_{r} \neq i_{r+1} \pm 1, \\
\left(y_{r+1}-y_{r}\right) e(\mathbf{i}), & \text { if } e \neq 2 \text { and } i_{r+1}=i_{r}+1, \\
\left(y_{r}-y_{r+1}\right) e(\mathbf{i}), & \text { if } e \neq 2 \text { and } i_{r+1}=i_{r}-1, \\
\left(y_{r+1}-y_{r}\right)\left(y_{r}-y_{r+1}\right) e(\mathbf{i}), & \text { if } e=2 \text { and } i_{r+1}=i_{r}+1\end{cases} \\
& \psi_{r} \psi_{r+1} \psi_{r} e(\mathbf{i})= \begin{cases}\left(\psi_{r+1} \psi_{r} \psi_{r+1}+1\right) e(\mathbf{i}), & \text { if } e \neq 2 \text { and } i_{r+2}=i_{r}=i_{r+1}-1, \\
\left(\psi_{r+1} \psi_{r} \psi_{r+1}-1\right) e(\mathbf{i}), & \text { if } e \neq 2 \text { and } i_{r+2}=i_{r}=i_{r+1}+1, \\
\left(\psi_{r+1} \psi_{r} \psi_{r+1}+y_{r}\right. & \\
\left.-2 y_{r+1}+y_{r+2}\right) e(\mathbf{i}), & \text { if } e=2 \text { and } i_{r+2}=i_{r}=i_{r+1}+1, \\
\psi_{r+1} \psi_{r} \psi_{r+1} e(\mathbf{i}), & \text { otherwise. }\end{cases}
\end{aligned}
$$


for $\mathbf{i}, \mathbf{j} \in I^{n}$ and all admissible $r$ and s. Moreover, $\mathscr{R}_{n}^{\Lambda}$ is naturally $\mathbb{Z}$-graded with degree function determined by

$$
\operatorname{deg} e(\mathbf{i})=0, \quad \operatorname{deg} y_{r}=2 \quad \text { and } \quad \operatorname{deg} \psi_{s} e(\mathbf{i})=-a_{i_{s}, i_{s+1}},
$$

for $1 \leq r \leq n, 1 \leq s<n$ and $\mathbf{i} \in I^{n}$.

To make the link between the cyclotomic Hecke algebra $\mathscr{H}_{n}$ and the quiver Hecke algebra $\mathscr{R}_{n}^{\Lambda}$ suppose that $R=K$ is a field of characteristic $p \geq 0$. Fix non-zero element $\xi$ of $K$ and let $e \in\{0,2,3,4, \ldots\}$ is the quantum characteristic of $\xi$. As noted after Definition 2.1] if the parameters $\mathbf{Q}$ are integral then the algebra $\mathscr{H}_{n}=\mathscr{H}_{n}(e, \boldsymbol{\kappa})$ is determined by $e$ and the multicharge $\kappa$. Let $\Lambda=\Lambda_{\kappa} \in X^{+}$be the unique positive weight such that

$$
\left(\Lambda, \alpha_{i}\right)=\#\left\{1 \leq l \leq \ell \mid \kappa_{l} \equiv i(\bmod e)\right\}, \quad \text { for all } i \in I .
$$

We define $\mathscr{H}_{n}^{\Lambda}=\mathscr{H}_{n}(e, \boldsymbol{\kappa})$, where $\Lambda=\Lambda_{\boldsymbol{\kappa}}$.

Next observe that (2.3) together with Theorem 2.2 implies that $\mathscr{H}_{n}^{\Lambda}$ decomposes into a direct sum of (simultaneous) generalized eigenspaces for the elements $L_{1}, \ldots, L_{n}$. Moreover, the possible eigenvalues for $L_{1}, \ldots, L_{n}$ are precisely the integers in $I$, if $\xi=1$, and otherwise they are belong to the set $\left\{\xi^{i} \mid i \in I\right\}$, if $\xi \neq 1$. Hence, the generalized eigenspaces for these elements are indexed by $I^{n}$. For each $\mathbf{i} \in I^{n}$ let $e(\mathbf{i})$ be the corresponding idempotent in $\mathscr{H}_{n}^{\Lambda}$ (or zero if the corresponding eigenspace is zero).

3.2. Theorem (Brundan-Kleshchev). Suppose that $R=K$ is a field, $\xi \in K$ as above, and that $\Lambda=\Lambda_{\kappa}$. Then there is an isomorphism of algebras $\mathscr{R}_{n}^{\Lambda} \cong \mathscr{H}_{n}^{\Lambda}$ which sends $e(\mathbf{i}) \mapsto e(\mathbf{i})$, for all $\mathbf{i} \in I^{n}$ and

$$
\begin{aligned}
& y_{r} \mapsto \begin{cases}\sum_{\mathbf{i} \in I^{n}}\left(1-\xi^{-i_{r}} L_{r}\right) e(\mathbf{i}), & \text { if } \xi \neq 1, \\
\sum_{\mathbf{i} \in I^{n}}\left(L_{r}-i_{r}\right) e(\mathbf{i}), & \text { if } \xi=1 .\end{cases} \\
& \psi_{s} \mapsto \sum_{\mathbf{i} \in I^{n}}\left(T_{r}+P_{r}(\mathbf{i})\right) Q_{r}(\mathbf{i})^{-1} e(\mathbf{i}),
\end{aligned}
$$

where $P_{r}(\mathbf{i}), Q_{r}(\mathbf{i}) \in R\left[y_{r}, y_{r+1}\right]$, for $1 \leq r \leq n$ and $1 \leq s<n$.

We abuse notation and identify the algebras $\mathscr{R}_{n}^{\Lambda}$ and $\mathscr{H}_{n}^{\Lambda}$ under this isomorphism. In particular, we will not distinguish between the homogeneous generators of $\mathscr{R}_{n}^{\Lambda}$ and their images in $\mathscr{H}_{n}^{\Lambda}$ under the isomorphism of Theorem 3.2.

The algebra $\mathscr{R}_{n}^{\Lambda} \cong \mathscr{H}_{n}^{\Lambda}$ has a unique anti-isomorphism $\star: \mathscr{R}_{n}^{\Lambda} \longrightarrow \mathscr{R}_{n}^{\Lambda} ; a \mapsto a^{\star}$ which fixes each of the homogeneous generators. We note that the automorphism $\star$ is, in general, not equal to the anti-automorphism $*$ which fixes each of the (non-homogeneous) generators of $\mathscr{H}_{n}^{\Lambda}$ in Definition 2.1

Until further notice fix a multipartition $\boldsymbol{\mu} \in \mathscr{P}_{n}$. If $i \in I$ then an $i$-node is a triple $(r, c, l) \in \mathbb{N}^{2} \times\{1,2, \ldots, \ell\}$ such that $i=c-r+\kappa_{l}(\bmod e)$. An $i$-node $A$ is an addable $i$-node of $\boldsymbol{\mu}$ if $A \notin[\boldsymbol{\mu}]$ and $[\boldsymbol{\mu}] \cup\{A\}$ is the diagram of a multipartition. Similarly, an $i$-node $B \in[\boldsymbol{\mu}]$ is a removable $i$-node of $\boldsymbol{\mu}$ if $[\boldsymbol{\mu}] \backslash\{B\}$ is the diagram of a multipartition. Given two nodes $A=(r, c, l)$ and $B=(s, d, m)$ then $A$ is below $B$, or $B$ is above $A$, if either $l>m$, or $l=m$ and $r>s$.

Following Brundan, Kleshchev and Wang, we make the following definitions. 
3.3. Definition (Brundan, Kleshchev and Wang [8] Defn. 3.5]). Suppose that $\boldsymbol{\mu} \in \mathscr{P}_{n}$ and that $A$ is a removable or addable $i$-node of $\boldsymbol{\mu}$, for some $i \in I$. Define integers

$$
d_{A}(\boldsymbol{\mu})=\#\left\{\begin{array}{c}
\text { addable } i \text {-nodes of } \boldsymbol{\mu} \\
\text { strictly below } A
\end{array}\right\}-\#\left\{\begin{array}{c}
\text { removable } i \text {-nodes of } \boldsymbol{\mu} \\
\text { strictly below } A
\end{array}\right\},
$$

and

$$
d^{A}(\boldsymbol{\mu})=\#\left\{\begin{array}{c}
\text { addable } i \text {-nodes of } \boldsymbol{\mu} \\
\text { strictly above } A
\end{array}\right\}-\#\left\{\begin{array}{c}
\text { removable } i \text {-nodes of } \boldsymbol{\mu} \\
\text { strictly above } A
\end{array}\right\} .
$$

If $\mathfrak{t}$ is a standard $\boldsymbol{\mu}$-tableau then its degree and codegree are defined inductively by setting $\operatorname{deg} \mathfrak{t}=0=\operatorname{codeg} \mathfrak{t}$, if $n=0$, and if $n>0$ then

$$
\operatorname{deg} \mathfrak{t}=\operatorname{deg} \mathfrak{t}_{n-1}+d_{A}(\boldsymbol{\nu}) \quad \text { and } \quad \operatorname{codeg} \mathfrak{t}=\operatorname{codeg} \mathfrak{t}_{n-1}+d^{A}(\boldsymbol{\nu})
$$

where $A=\mathfrak{t}^{-1}(n)$ and $\boldsymbol{\nu}=\operatorname{Shape}\left(\mathfrak{t}_{n-1}\right)$.

If $\mathfrak{t}$ is a standard tableau define $\operatorname{res}(\mathfrak{t})=\left(\operatorname{res}_{\mathfrak{t}}(1), \ldots, \operatorname{res}_{\mathfrak{t}}(n)\right) \in I^{n}$, where $\operatorname{res}_{\mathfrak{t}}(k)=$ $c-r+\kappa_{l}(\bmod e)$ if $k$ appears in row $r$ and column $c$ of $\mathfrak{t}^{(l)}$, for $1 \leq k \leq n$. This definition is compatible with our previous definition of $\operatorname{cont}_{t}(k)$ in the sense that if $R=K$ then $\operatorname{cont}_{\mathfrak{t}}(k) \equiv \operatorname{res}_{\mathfrak{t}}(k)(\bmod e)$, if $\xi=1$, and $\operatorname{cont}_{\mathfrak{t}}(k)=\xi^{\operatorname{res}_{\mathfrak{t}}(k)}$, if $\xi \neq 1$.

3.4. Definition ( [13, Definitions 4.9, 5.1 and 6.9]). Suppose that $\boldsymbol{\mu} \in \mathscr{P}_{n}$. Let $\mathbf{i}_{\mu}=$ $\operatorname{res}\left(\mathfrak{t}_{\boldsymbol{\mu}^{\prime}}\right)$ and $\mathbf{i}^{\mu}=\operatorname{res}\left(\mathfrak{t}^{\boldsymbol{\mu}}\right)$ and set $e_{\boldsymbol{\mu}}=e\left(\mathbf{i}^{\boldsymbol{\mu}}\right)$ and $e_{\boldsymbol{\mu}}^{\prime}=e\left(\mathbf{i}_{\boldsymbol{\mu}}\right)$ and define

$$
y_{\boldsymbol{\mu}}=y_{1}^{d_{1}} \ldots y_{n}^{d_{n}} \text { and } y_{\boldsymbol{\mu}}^{\prime}=y_{1}^{d_{1}^{\prime}} \ldots y_{n}^{d_{n}^{\prime}} \text {, }
$$

where $d_{m}=d_{A_{m}}\left(\boldsymbol{\mu}_{m}\right), d_{m}^{\prime}=d^{A_{m}^{\prime}}\left(\boldsymbol{\mu}_{m}^{\prime}\right), \boldsymbol{\mu}_{m}=$ Shape $\left(\mathfrak{t}_{m}^{\mu}\right), \boldsymbol{\mu}_{m}^{\prime}=\operatorname{Shape}\left(\left(\mathfrak{t}_{\boldsymbol{\mu}^{\prime}}\right)_{m}\right)$, and $A_{m}$ and $A_{m}^{\prime}$ are the nodes such that $\mathfrak{t}^{\mu}\left(A_{m}\right)=m$ and $\mathfrak{t}_{\mu^{\prime}}\left(A_{m}^{\prime}\right)=m$.

For the rest of this paper, fix a reduced expression $d(\mathfrak{u})=s_{i_{1}} \ldots s_{i_{k}}$ for each row standard $\boldsymbol{\mu}$-tableau $\mathfrak{u}$ and set $\psi_{d(\mathfrak{u})}=\psi_{i_{1}} \ldots \psi_{i_{k}}$. Suppose that $(\mathfrak{s}, \mathfrak{t}) \in \operatorname{Std}^{2}(\boldsymbol{\mu})$ and define $\psi_{\mathfrak{s t}}=\psi_{d(\mathfrak{s})}^{\star} e_{\boldsymbol{\mu}} y_{\boldsymbol{\mu}} \psi_{d(\mathfrak{t})}$ and $\psi_{\mathfrak{s t}}^{\prime}=\psi_{d(\mathfrak{s})}^{\star} e_{\boldsymbol{\mu}}^{\prime} y_{\boldsymbol{\mu}}^{\prime} \psi_{d(\mathfrak{t})}$.

We warn the reader that, in general, the elements $\psi_{d(\mathfrak{s})}, \psi_{d(\mathfrak{t})}, \psi_{\mathfrak{s t}}$ and $\psi_{\mathfrak{s t}}^{\prime}$ all depend upon the choices of reduced expression for $d(\mathfrak{s})$ and $d(\mathfrak{t})$ that we have fixed, once and for all, in Definition 3.4 See [13, Example 5.6] for an explicit example.

The following result can be viewed as a graded analogue of Theorem 2.2

3.5. Theorem (Hu-Mathas [13, Theorems 5.8 and 6.11]).

a) $\left\{\psi_{\mathfrak{s t}} \mid(\mathfrak{s}, \mathfrak{t}) \in \operatorname{Std}^{2}\left(\mathscr{P}_{n}\right)\right\}$ is a graded cellular basis of $\mathscr{H}_{n}^{\Lambda}$.

b) $\left\{\psi_{\mathfrak{s t}}^{\prime} \mid(\mathfrak{s}, \mathfrak{t}) \in \operatorname{Std}^{2}\left(\mathscr{P}_{n}\right)\right\}$ is a graded cellular basis of $\mathscr{H}_{n}^{\Lambda}$.

In particular, if $(\mathfrak{s}, \mathfrak{t}) \in \operatorname{Std}^{2}\left(\mathscr{P}_{n}\right)$ then

$$
\operatorname{deg} \psi_{\mathfrak{s t}}=\operatorname{deg} \mathfrak{s}+\operatorname{deg} \mathfrak{t} \quad \text { and } \quad \operatorname{deg} \psi_{\mathfrak{s t}}^{\prime}=\operatorname{codeg} \mathfrak{s}^{\prime}+\operatorname{codeg} \mathfrak{t}^{\prime} .
$$

Graded cellular algebras were introduced in [13]. They are a natural extension of Graham and Lehrer's [12] definition of a cellular algebra to the graded setting.

We close this section by extending results in the last subsection about strong dominance to the $\psi$ and $\psi^{\prime}$-bases.

3.6. Lemma. Suppose that $R=K$ and $\boldsymbol{\mu} \in \mathscr{P}_{n}$. Then

$$
\psi_{\mathfrak{t}^{\mu} \mathfrak{t}^{\mu}}=\sum_{(\mathfrak{u}, \mathfrak{v})} r_{\left.\mathfrak{t}^{\mu}, \mathfrak{t}^{\mu}\right)} r_{\mathfrak{u v}} m_{\mathfrak{u v}} \text { and } \psi_{\mathfrak{t}^{\mu} \mathfrak{t}^{\mu}}^{\prime}=\sum_{(\mathfrak{u}, \mathfrak{v})} \sum_{\left(\mathfrak{t}^{\mu}, \mathfrak{t}^{\mu}\right)} s_{\mathfrak{u v}} n_{\mathfrak{u v}},
$$

for some $r_{\mathfrak{u v}}, s_{\mathfrak{u v}} \in K$ such that $r_{\mathfrak{t}^{\mu} \mathfrak{t}^{\mu}} \neq 0$ and $\mathfrak{s}_{\mathfrak{t}^{\mu} \mathfrak{t}^{\mu}} \neq 0$. 
Proof. As in [13, Definition 4.3] we fix a modular system $(\mathcal{O}, \mathcal{K}, K)$ with parameters $v \in \mathcal{O}$ and $\mathbf{Q}^{\mathcal{O}}=\left(Q_{1}^{\mathcal{O}}, \ldots, Q_{\ell}^{\mathcal{O}}\right) \in \mathcal{O}^{\ell}$ such that $\mathscr{H}_{n}^{\mathcal{K}}=\mathscr{H}_{n}^{\mathcal{O}} \otimes_{\mathcal{O}} \mathcal{K}$ is split semisimple and $\mathscr{H}_{n}^{K} \cong \mathscr{H}_{n}^{\mathcal{O}} \otimes_{\mathcal{O}} K$, where $\mathscr{H}_{n}^{\mathcal{O}}=\mathscr{H}_{n}^{\mathcal{O}}\left(v, \mathbf{Q}^{\mathcal{O}}\right)$. By [13] Defn. 4.12], there exist elements $y_{\boldsymbol{\mu}}^{\mathcal{O}}$ and $e_{\boldsymbol{\mu}}^{\mathcal{O}}$ in $\mathscr{H}_{n}^{\mathcal{O}}$ such that $e_{\boldsymbol{\mu}} y_{\boldsymbol{\mu}}=e_{\boldsymbol{\mu}}^{\mathcal{O}} y_{\boldsymbol{\mu}}^{\mathcal{O}} \otimes_{\mathcal{O}} 1_{K}$. Moreover, by [13 Lemma 4.13] there exist scalars $r_{\mathfrak{s}} \in \mathcal{K}$ such that in $\mathscr{H}_{n}^{\mathcal{K}}$

$$
e_{\boldsymbol{\mu}}^{\mathcal{O}} y_{\boldsymbol{\mu}}^{\mathcal{O}}=\sum_{\substack{\mathfrak{s} \unrhd \mathfrak{t}^{\mu} \\ \operatorname{res}(\mathfrak{s})=\mathbf{i}^{\mu}}} r_{\mathfrak{s}} f_{\mathfrak{s s}} .
$$

Therefore, by Theorem 2.13, there exist $r_{\mathfrak{u v}}^{\mathcal{K}} \in \mathcal{K}$ such that

$$
e_{\boldsymbol{\mu}}^{\mathcal{O}} y_{\boldsymbol{\mu}}^{\mathcal{O}}=\sum_{(\mathfrak{u}, \mathfrak{v})} \sum_{\left(\mathfrak{t}^{\mu}, \mathfrak{t}^{\mu}\right)} r_{\mathfrak{u v}}^{\mathcal{K}} m_{\mathfrak{u v}} .
$$

However, $e_{\boldsymbol{\mu}}^{\mathcal{O}} y_{\boldsymbol{\mu}}^{\mathcal{O}} \in \mathscr{H}_{n}^{\mathcal{O}}$ and $m_{\mathfrak{u v}} \in \mathscr{H}_{n}^{\mathcal{O}}$ for all $(\mathfrak{u}, \mathfrak{v}) \in \operatorname{Std}^{2}\left(\mathscr{P}_{n}\right)$ so, in fact, $r_{\mathfrak{u v}}^{\mathcal{K}} \in \mathcal{O}$. Hence, we can reduce this equation modulo the maximal ideal of $\mathcal{O}$ to write $e_{\boldsymbol{\mu}} y_{\boldsymbol{\mu}}=\psi_{\mathbf{t}^{\mu} \boldsymbol{t}^{\mu}}$ in the required form.

We leave the proof of the formula for $\psi_{\mathfrak{t}^{\mu} \boldsymbol{t}^{\mu}}^{\prime}$ to the reader. It is proved in exactly same way except that [13, Lemma 6.5] is used in place of [13, Lemma 4.13].

3.7. Theorem. Suppose that $R=K$ and $(\mathfrak{s}, \mathfrak{t}) \in \operatorname{Std}^{2}\left(\mathscr{P}_{n}\right)$. Then

$$
\begin{aligned}
\psi_{\mathfrak{s t}} & =\sum_{(\mathfrak{u}, \mathfrak{v}) \mathbf{\unrhd}(\mathfrak{s}, \mathfrak{t})} a_{\mathfrak{u v}} m_{\mathfrak{u v}}, & m_{\mathfrak{s t}} & =\sum_{(\mathfrak{u}, \mathfrak{v}) \mathbf{\unrhd}(\mathfrak{s}, \mathfrak{t})} c_{\mathfrak{u v}} \psi_{\mathfrak{u v},}, \\
\psi_{\mathfrak{s t}}^{\prime} & =\sum_{(\mathfrak{u}, \mathfrak{v}) \mathbf{\unrhd}(\mathfrak{s}, \mathfrak{t})} b_{\mathfrak{u v}} n_{\mathfrak{u v}}, & n_{\mathfrak{s t}} & =\sum_{(\mathfrak{u}, \mathfrak{v}) \mathbf{\unrhd}(\mathfrak{s}, \mathfrak{t})} d_{\mathfrak{u v}} \psi_{\mathfrak{u v}}^{\prime}
\end{aligned}
$$

for some scalars $a_{\mathfrak{u v}}, b_{\mathfrak{u v}}, c_{\mathfrak{u v}}$ and $d_{\mathfrak{u v}}$ in $K$ such that $a_{\mathfrak{s t}}, b_{\mathfrak{s t}}, c_{\mathfrak{s t}}$ and $d_{\mathfrak{s t}}$ are all non-zero.

Proof. For convenience, let $\mathbf{i}^{s}=\operatorname{res}(\mathfrak{s})$ and $\mathbf{i}^{\mathfrak{t}}=\operatorname{res}(\mathfrak{t})$. By Theorem 3.2 and Definition 3.4 if $d(\mathfrak{t})=s_{i_{1}} \ldots s_{i_{k}}$ is reduced then

$$
\psi_{\mathfrak{t}^{\mu} \mathfrak{t}}=\psi_{\mathfrak{t}^{\mu} \mathfrak{t} \mu}\left(T_{i_{1}}+P_{i_{1}}\left(\mathbf{i}_{1}\right)\right) Q_{i_{1}}\left(\mathbf{i}_{1}\right)^{-1} e\left(\mathbf{i}_{1}\right) \ldots \ldots\left(T_{i_{k}}+P_{i_{k}}\left(\mathbf{i}_{k}\right)\right) Q_{i_{k}}\left(\mathbf{i}_{k}\right)^{-1} e\left(\mathbf{i}_{k}\right),
$$

where $\mathbf{i}_{1}=\left(\mathbf{i}^{\mu}\right)^{s_{i_{1}}}$ and $\mathbf{i}_{j}=\mathbf{i}_{j-1}^{s_{i_{j}}}$ for all $2 \leq j \leq k$ with $\mathfrak{S}_{n}$ acting on $I^{n}$ from the right in the natural way. (Thus, $\mathbf{i}_{k}=\mathbf{i}^{\mathrm{t}}$.) Using the relations we can rewrite the expression for $\psi_{d(\mathfrak{t})} e\left(\mathbf{i}^{\mathfrak{t}}\right)$ as $r T_{d(\mathfrak{t})} e\left(\mathbf{i}^{\mathfrak{t}}\right)$ plus a linear combination of terms of the form $L T_{u}$, where $r \in K$ is non-zero, $u<d(\mathfrak{t})$ and $L \in\left\langle L_{1}, \ldots, L_{n}\right\rangle$. By Lemma 3.6 and Corollary 2.13, $\psi_{\mathfrak{t}^{\mu} \mathfrak{t}^{\mu}} L$ can be written as linear combinations of elements of form $m_{\mathfrak{u v}}$ with $(\mathfrak{u}, \mathfrak{v}) \unrhd\left(\mathfrak{t}^{\boldsymbol{\mu}}, \mathfrak{t}^{\boldsymbol{\mu}}\right)$. Therefore, applying Lemma 2.10 to the elements $m_{\mathfrak{u v}} T_{u}$ shows that $\psi_{\mathfrak{t}^{\mu_{\mathfrak{t}}}}$ can be written as a linear combination of elements $m_{\mathfrak{u} \mathfrak{v}}$ with $(\mathfrak{u}, \mathfrak{v}) \geq\left(\mathfrak{t}^{\mu}, \mathfrak{t}\right)$. Using the same argument to act with $e\left(\mathbf{i}^{\mathfrak{s}}\right) \psi_{d(\mathfrak{s})}^{\star}$ from the left shows that $\psi_{\mathfrak{s t}}$ can be written in the required form. Arguing by induction on the strong dominance order $\square$ we can now invert this equation to show that $m_{\mathfrak{s t}}$ is a linear combination of elements $\psi_{\mathfrak{u v}}$ with $(\mathfrak{u}, \mathfrak{v}) \geq(\mathfrak{s}, \mathfrak{t})$.

The other equations can be proved similarly.

3.8. Corollary. Suppose that $R=K$ and $(\mathfrak{s}, \mathfrak{t}),(\mathfrak{u}, \mathfrak{v}) \in \operatorname{Std}^{2}\left(\mathscr{P}_{n}\right)$. Then $\psi_{\mathfrak{s t}} \psi_{\mathfrak{u v}}^{\prime} \neq 0$ only if $\mathfrak{u}^{\prime} \unrhd \mathfrak{t}$ and $\psi_{\mathfrak{u v}}^{\prime} \psi_{\mathfrak{s t}} \neq 0$ only if $\mathfrak{v}^{\prime} \unrhd \mathfrak{s}$.

Proof. By Theorem 3.7 there exist scalars $a_{\mathfrak{c d}}$ and $b_{\mathfrak{a} \mathfrak{b}}$ such that

$$
\psi_{\mathfrak{u} \mathfrak{v}}^{\prime} \psi_{\mathfrak{s t}}=\left(\sum_{(\mathfrak{c}, \mathfrak{d}) \geq(\mathfrak{u}, \mathfrak{v})} b_{\mathfrak{c o}} n_{\mathfrak{c} \mathfrak{d}}\right)\left(\sum_{(\mathfrak{a}, \mathfrak{b}) \geq(\mathfrak{s , \mathfrak { t } )}} a_{\mathfrak{a} \mathfrak{b}} m_{\mathfrak{a} \mathfrak{b}}\right) .
$$


Therefore, $\psi_{\mathfrak{u} \mathfrak{v}}^{\prime} \psi_{\mathfrak{s t}} \neq 0$ only if there exist tableaux $\mathfrak{a}, \mathfrak{b}, \mathfrak{c}$ and $\mathfrak{d}$ such that $n_{\mathfrak{c} \mathfrak{d}} m_{\mathfrak{a} \mathfrak{b}} \neq 0$ and $(\mathfrak{c}, \mathfrak{d}) \unrhd(\mathfrak{u}, \mathfrak{v})$ and $(\mathfrak{a}, \mathfrak{b}) \geq(\mathfrak{s}, \mathfrak{t})$. By Corollary 2.13 this happens only if $\mathfrak{d}^{\prime} \unrhd \mathfrak{a}$. Therefore, $\mathfrak{v}^{\prime} \unrhd \mathfrak{d}^{\prime} \unrhd \mathfrak{a} \unrhd \mathfrak{s}$ as required. The result for $\psi_{\mathfrak{s t}} \psi_{\mathfrak{u v}}^{\prime}$ now follows by applying the graded involution $\star$.

We leave the following result as an exercise for the reader. It follows easily using Theorem 3.2 and Corollary 2.13 .

3.9. Corollary. Suppose that $R=K$ and let $(\mathfrak{s}, \mathfrak{t}) \in \operatorname{Std}^{2}\left(\mathscr{P}_{n}\right)$. Then there exist scalars $a_{\mathfrak{u v}}, b_{\mathfrak{u v}} \in K$ such that

$$
\psi_{\mathfrak{s t}} y_{r}=\sum_{(\mathfrak{u}, \mathfrak{v}) \backslash(\mathfrak{s}, \mathfrak{t})} a_{\mathfrak{u v}} \psi_{\mathfrak{u v}} \quad \text { and } \quad \psi_{\mathfrak{s t}}^{\prime} y_{r}=\sum_{(\mathfrak{a}, \mathfrak{b}) \backslash(\mathfrak{s}, \mathfrak{t})} b_{\mathfrak{a} \mathfrak{b}} \psi_{\mathfrak{a} \mathfrak{b}}^{\prime}
$$

Moreover, $a_{\mathfrak{u} \mathfrak{v}}$ and $b_{\mathfrak{a} \mathfrak{b}}$ are non-zero only when $\operatorname{res}(\mathfrak{u})=\operatorname{res}(\mathfrak{s}), \operatorname{res}(\mathfrak{v})=\operatorname{res}(\mathfrak{t}), \operatorname{res}(\mathfrak{a})=$ $\operatorname{res}(\mathfrak{s}), \operatorname{res}(\mathfrak{b})=\operatorname{res}(\mathfrak{t})$ and $\operatorname{deg} \mathfrak{u}+\operatorname{deg} \mathfrak{v}=\operatorname{deg} \mathfrak{s}+\operatorname{deg} \mathfrak{t}+2, \operatorname{codeg} \mathfrak{a}^{\prime}+\operatorname{codeg} \mathfrak{b}^{\prime}=$ $\operatorname{codeg} \mathfrak{s}^{\prime}+\operatorname{codeg} \mathfrak{t}^{\prime}+2$.

\section{GRAded induction AND GRAded Specht Modules}

Before starting the proof of our Main Theorem we recall the facts that we need about $\mathbb{Z}$-graded algebras and the construction of the graded Specht modules and their duals.

Suppose that $A=\bigoplus_{k \in \mathbb{Z}} A_{k}$ is a $\mathbb{Z}$-graded algebra. If $a \in A_{k}$ then $a$ is homogeneous of degree $\operatorname{deg} a=k$. If $M=\bigoplus_{k} M_{k}$ is a graded $A$-module let $M\langle s\rangle$ be the graded $R$-module obtained by shifting the grading on $M$ upwards by $s$; that is, $M\langle s\rangle_{k}=M_{k-s}$, for $k \in \mathbb{Z}$. Let Mod- $A$ be the category of finite dimensional graded (right) $A$-module with homomorphisms being the degree preserving maps (of degree zero). If $A$ has a degree preserving anti-involution $\star$ then the contragredient dual of $M$ is the graded $A$-module

$$
M^{\circledast}=\bigoplus_{d \in \mathbb{Z}} \operatorname{Hom}_{A}(M\langle d\rangle, K)
$$

where the action of $A$ is given by $(f a)(m)=f\left(m a^{\star}\right)$, for all $f \in M^{\circledast}, a \in A$ and $m \in M$.

4.1. Graded Specht modules. Following the standard construction from the theory of (graded) cellular algebras, the two graded cellular bases of Theorem 3.5 define graded cell modules for $\mathscr{H}_{n}^{\Lambda}$. More explicitly, for each multipartition $\boldsymbol{\mu} \in \mathscr{P}_{n}$ the graded Specht module $S^{\mu}$ and the graded dual Specht module $S_{\mu}$ are the graded $\mathscr{H}_{n}^{\Lambda}$-modules such that

$$
S^{\boldsymbol{\mu}}\left\langle\operatorname{deg} \mathfrak{t}^{\boldsymbol{\mu}}\right\rangle=\left(\psi_{\mathfrak{t}^{\mu} \mathfrak{t}^{\mu}}+\mathscr{H}_{\boldsymbol{\mu}}\right) \mathscr{H}_{n}^{\Lambda} \quad \text { and } \quad S_{\boldsymbol{\mu}}\left\langle\operatorname{codeg} \mathfrak{t}_{\boldsymbol{\mu}^{\prime}}\right\rangle=\left(\psi_{\mathfrak{t}^{\mu} \boldsymbol{t}^{\mu}}^{\prime}+\mathscr{H}_{\boldsymbol{\mu}}^{\prime}\right) \mathscr{H}_{n}^{\Lambda},
$$

where $\mathscr{H}_{\boldsymbol{\mu}}$ is the two-sided ideal of $\mathscr{H}_{n}^{\Lambda}$ spanned by the elements $\psi_{\mathfrak{u v}}$, where $(\mathfrak{u}, \mathfrak{v}) \in$ $\operatorname{Std}^{2}(\boldsymbol{\nu}), \boldsymbol{\nu} \triangleright \boldsymbol{\mu}$ and $\mathscr{H}_{\boldsymbol{\mu}}^{\prime}$ is spanned by the $\psi_{\mathfrak{u v}}^{\prime}$, for $(\mathfrak{u}, \mathfrak{v}) \in \operatorname{Std}^{2}(\boldsymbol{\nu})$ with $\boldsymbol{\nu} \triangleright \boldsymbol{\mu}$. Thus, $S^{\mu}$ has a natural basis $\left\{\psi_{\mathfrak{t}} \mid \mathfrak{t} \in \operatorname{Std}(\boldsymbol{\mu})\right\}$, and $S_{\boldsymbol{\mu}}$ has a basis $\left\{\psi_{\mathfrak{t}}^{\prime} \mid \mathfrak{t} \in \operatorname{Std}(\boldsymbol{\mu})\right\}$, where the action on both modules in induced by the action of $\mathscr{H}_{n}^{\Lambda}$ upon the $\psi$ and $\psi^{\prime}$ bases of $\mathscr{H}_{n}^{\Lambda}$, respectively, and $\operatorname{deg} \psi_{\mathfrak{t}}=\operatorname{deg} \mathfrak{t}, \operatorname{deg} \psi_{\mathfrak{t}}^{\prime}=\operatorname{codeg} \mathfrak{t}^{\prime}$, for $\mathfrak{t} \in \operatorname{Std}(\boldsymbol{\mu})$. In particular, by [13, Prop. 6.19], $S^{\mu}$ is isomorphic to the graded Specht module defined by Brundan, Kleshchev and Wang $[\mathbf{8}]$. See [13, $\$ 2]$ for more details.

To explain the relationship between the graded Specht module and its dual we need to recall the description of the blocks of $\mathscr{H}_{n}^{\Lambda}$. Let $\beta \in Q_{+}$be a positive root with 
$\sum_{i \in I}\left(\Lambda_{i}, \beta\right)=n$ and set $I^{\beta}=\left\{\mathbf{i} \in I^{n} \mid \alpha_{i_{1}}+\cdots+\alpha_{i_{n}}=\beta\right\}$ and $e_{\beta}=\sum_{\mathbf{i} \in I^{\beta}} e(\mathbf{i})$. By [17, Theorem 2.11] and [4, Theorem 1],

$$
\mathscr{H}_{n}^{\Lambda}=\bigoplus_{\beta \in Q_{+}, I^{\beta} \neq \emptyset} \mathscr{H}_{\beta}^{\Lambda}, \quad \text { where } \mathscr{H}_{\beta}^{\Lambda}=e_{\beta} \mathscr{H}_{n}^{\Lambda},
$$

where $\mathscr{H}_{\beta}^{\Lambda}$ is an indecomposable two-sided ideal of $\mathscr{H}_{n}^{\Lambda}$ whenever $e_{\beta} \neq 0$. Following Brundan and Kleshchev [8, (3.4)], if $\beta \in Q_{+}$with $e_{\beta} \neq 0$ then define the defect of $\mathscr{H}_{\beta}^{\Lambda}$ by

$$
\operatorname{def} \beta=(\Lambda, \beta)-\frac{1}{2}(\beta, \beta) .
$$

Then $\operatorname{def} \beta \geq 0$. Let $\mathscr{P}_{\beta}=\left\{\boldsymbol{\mu} \in \mathscr{P}_{n} \mid \mathbf{i}^{\mu} \in I^{\beta}\right\}$ and suppose that $\boldsymbol{\mu} \in \mathscr{P}_{\beta}$. Then, by [13, Prop. 6.19],

$$
S^{\boldsymbol{\mu}} \cong S_{\boldsymbol{\mu}^{\prime}}^{\circledast}\langle\operatorname{def} \beta\rangle,
$$

where $S_{\boldsymbol{\mu}^{\prime}}^{\circledast}$ is the graded dual of $S_{\boldsymbol{\mu}^{\prime}}$. This justifies calling $S_{\boldsymbol{\mu}}$ a graded dual Specht module. For the rest of this section we fix $\beta \in Q_{+}$and $\boldsymbol{\mu} \in \mathscr{P}_{\beta}$ such that $e_{\beta} \neq 0$.

To prove our Main Theorem we need to compute $i$-Ind $S^{\mu}$, for $i \in I$. To do this we will use another construction of the graded Specht modules which, up to shift, realizes them as submodules of $\mathscr{H}_{n}^{\Lambda}$. Recall that $\mathfrak{t}^{\mu}$ is the unique standard $\boldsymbol{\mu}$-tableau such that $\mathfrak{t}^{\mu} \unrhd \mathfrak{t}$ for all $\mathfrak{t} \in \operatorname{Std}(\boldsymbol{\mu})$. Let $\mathfrak{t}_{\boldsymbol{\mu}}$ be the unique standard $\boldsymbol{\mu}$-tableau such that $\mathfrak{t} \unrhd \mathfrak{t}_{\boldsymbol{\mu}}$ for all $t \in \operatorname{Std}(\boldsymbol{\mu})$. Then $\mathfrak{t}_{\boldsymbol{\mu}}$ is the standard $\boldsymbol{\mu}$-tableau which has the numbers $1,2, \ldots, n$ entered in order down its columns in the components from right to left. Equivalently, $\mathfrak{t}_{\mu}$ is the tableau conjugate to $\mathfrak{t}^{\boldsymbol{\mu}^{\prime}}$. Define $w_{\boldsymbol{\mu}}=d\left(\mathfrak{t}_{\boldsymbol{\mu}}\right) \in \mathfrak{S}_{n}$. It is easy to check that $w_{\boldsymbol{\mu}}^{-1}=w_{\boldsymbol{\mu}^{\prime}}=d\left(\mathfrak{t}_{\boldsymbol{\mu}^{\prime}}\right)$.

4.1. Definition. Suppose that $\boldsymbol{\mu} \in \mathscr{P}_{\beta}$, for $\beta \in Q_{+}$. Define $z_{\boldsymbol{\mu}}=y_{\boldsymbol{\mu}^{\prime}}^{\prime} \psi_{w_{\boldsymbol{\mu}^{\prime}}} e_{\boldsymbol{\mu}} y_{\boldsymbol{\mu}}$.

Consulting the definitions, $z_{\mu}=\psi_{\mathfrak{t}^{\prime} \mathfrak{t}_{\mu^{\prime}}}^{\prime} \psi_{\mathfrak{t}^{\mu} \mathfrak{t}^{\mu}}=\psi_{\mathfrak{t}^{\mu^{\prime} \mathfrak{t}^{\prime}}}^{\prime} \psi_{\mathfrak{t}_{\mu} \mathfrak{t}^{\mu}}$. The connection between these elements and the graded Specht modules is the following.

4.2. Lemma ([13, §6.4]). Suppose that $\boldsymbol{\mu} \in \mathscr{P}_{\beta}$. Then, as graded $\mathscr{H}_{n}^{\Lambda}$-modules,

$$
S^{\boldsymbol{\mu}}\left\langle\operatorname{def} \beta+\operatorname{codeg} \mathfrak{t}_{\boldsymbol{\mu}}\right\rangle \cong z_{\boldsymbol{\mu}} \mathscr{H}_{n}^{\Lambda} \quad \text { and } \quad S_{\boldsymbol{\mu}^{\prime}}\left\langle\operatorname{def} \beta+\operatorname{deg} \mathfrak{t}^{\boldsymbol{\mu}}\right\rangle \cong z_{\boldsymbol{\mu}}^{\star} \mathscr{H}_{n}^{\Lambda} \text {. }
$$

Hence, to determine $i$-Ind $S^{\mu}$ it suffices to describe the $\mathscr{H}_{n+1}^{\Lambda}$-module $i$-Ind $z_{\mu} \mathscr{H}_{n}^{\Lambda}$. To do this we adapt ideas which Ryom-Hansen [26] used to describe the induced Specht modules of $\mathscr{H}_{\xi}\left(\mathfrak{S}_{n}\right)$.

4.2. Graded induction of Specht modules. We are now ready to prove our main theorem. We start by describing the $i$-induction functors for $\mathscr{H}_{n}^{\Lambda}$ more explicitly.

Recall that $I=\mathbb{Z} / e \mathbb{Z}$. For each $i \in I$ define

$$
e_{i, n}=\sum_{\mathbf{j} \in I^{n}} e(\mathbf{j} \vee i) \in \mathscr{H}_{n+1}^{\Lambda} .
$$

Then $\sum_{i \in I} e_{i, n}=\sum_{\mathbf{i} \in I^{n+1}} e(\mathbf{i})$ is the identity element of $\mathscr{H}_{n+1}^{\Lambda} \cong \mathscr{R}_{n+1}^{\Lambda}$. Let Mod- $\mathscr{H}_{n}^{\Lambda}$ be the category of finite dimensional graded $\mathscr{H}_{n}^{\Lambda}$-modules, with morphisms being $\mathscr{H}_{n}^{\Lambda}$ module homomorphisms of degree zero.

4.3. Lemma. Suppose that $R=K$ is field and that $i \in I$. Then there is a (non-unital) embedding of graded algebras $\mathscr{H}_{n}^{\Lambda} \hookrightarrow \mathscr{H}_{n+1}^{\Lambda}$ given by

$$
e(\mathbf{j}) \mapsto e(\mathbf{j} \vee i), \quad y_{r} \mapsto e_{i, n} y_{r} \quad \text { and } \quad \psi_{s} \mapsto e_{i, n} \psi_{s},
$$

for $\mathbf{j} \in I^{n}, 1 \leq r \leq n$ and $1 \leq s<n$. This map induces an exact functor

$$
F_{i}: \operatorname{Mod}-\mathscr{H}_{n}^{\Lambda} \longrightarrow \operatorname{Mod}-\mathscr{H}_{n+1}^{\Lambda} ; M \mapsto M \otimes_{\mathscr{H}_{n}^{\Lambda}} e_{i, n} \mathscr{H}_{n+1}^{\Lambda} \text {. }
$$


Moreover, $F_{i}=i-\operatorname{Ind}_{\mathscr{H}_{n}^{\Lambda}}^{\mathscr{H}_{n}^{\Lambda}}$ is the graded induction functor from Mod- $\mathscr{H}_{n}^{\Lambda}$ to Mod- $\mathscr{H}_{n+1}^{\Lambda}$.

Proof. The images of the homogeneous generators of $\mathscr{H}_{n}^{\Lambda}$ under this embedding commute with $e_{i, n}$, which implies that this defines a non-unital degree preserving homomorphism from $\mathscr{H}_{n}^{\Lambda}$ to $\mathscr{H}_{n+1}^{\Lambda}$. This map is an embedding by Theorem 3.2 The remaining claims follow because $e_{i, n}$ is idempotent and $\sum_{i \in I} e_{i, n}$ is the identity element of $\mathscr{H}_{n+1}^{\Lambda}$.

We now fix a multipartition $\boldsymbol{\mu} \in \mathscr{P}_{n}$ and introduce the notation that we need to prove our main result. Let $A_{1}, \ldots, A_{z}$ be the addable $i$-nodes of $\boldsymbol{\mu}$ ordered so that $A_{i+1}$ is below $A_{i}$, for $1 \leq i<z$. Finally, let $\boldsymbol{\alpha}_{i}$ be the multipartition of $n+1$ obtained by adding $A_{i}$ to $\boldsymbol{\mu}$, so that $\left[\boldsymbol{\alpha}_{i}\right]=[\boldsymbol{\mu}] \cup\left\{A_{i}\right\}$, for $1 \leq i \leq Z$. Then $\boldsymbol{\alpha}_{1} \triangleright \boldsymbol{\alpha}_{2} \triangleright \cdots \triangleright \boldsymbol{\alpha}_{z}$ because we arranged the nodes $A_{1}, \ldots, A_{z}$ in "downwards order". For notational convenience in what follows we set $\boldsymbol{\alpha}=\boldsymbol{\alpha}_{1}$ and $\boldsymbol{\omega}=\boldsymbol{\alpha}_{z}$.

The two extremal multipartitions $\boldsymbol{\alpha}$ and $\boldsymbol{\omega}$ will be particularly important in what follows: $\boldsymbol{\alpha}$ is the most dominant multipartition obtained from $\boldsymbol{\mu}$ by adding an $i$-node and $\boldsymbol{\omega}$ is the least dominant such multipartition. Define $z_{\boldsymbol{\mu} \uparrow i}=e_{i, n} z_{\boldsymbol{\mu}}$ to be the image of $z_{\boldsymbol{\mu}}$ under the algebra embedding of Lemma 4.3 . Then by Definition 3.4 and Definition 4.1

$$
z_{\boldsymbol{\mu} \uparrow i}=e_{i, n} y_{\boldsymbol{\mu}^{\prime}}^{\prime} \psi_{w_{\boldsymbol{\mu}^{\prime}}} e_{\boldsymbol{\mu}} y_{\boldsymbol{\mu}} e_{i, n} .
$$

Therefore, by Lemma 4.2 and Lemma 4.3, we have the following.

4.4. Lemma. Suppose that $\boldsymbol{\mu} \in \mathscr{P}_{\beta}$ and $i \in I$. Then there is an isomorphism of graded $\mathscr{H}_{n+1}^{\Lambda}$-modules,

$$
i \text {-Ind } S^{\boldsymbol{\mu}}\left\langle\operatorname{def} \beta+\operatorname{codeg} \mathfrak{t}_{\boldsymbol{\mu}}\right\rangle \cong z_{\boldsymbol{\mu} \uparrow i} \mathscr{H}_{n+1}^{\Lambda} .
$$

To prove our main theorem we show that $z_{\boldsymbol{\mu} \uparrow i} \mathscr{H}_{n+1}^{\Lambda}$ has a filtration by graded Specht modules. For each $1 \leq k \leq z$, define $\mathfrak{t}_{\boldsymbol{\mu}}^{\boldsymbol{\alpha}_{k}}$ to be the unique standard $\alpha_{k}$-tableau such that $\left(\mathfrak{t}_{\boldsymbol{\mu}}^{\boldsymbol{\alpha}_{k}}\right)_{n}=\mathfrak{t}_{\boldsymbol{\mu}}$.

4.5. Proposition. Suppose that $\boldsymbol{\mu} \in \mathscr{P}_{\beta}$ and $i \in I$. Then

$$
\mathcal{B}_{\boldsymbol{\mu}, i}=\left\{e_{i, n} y_{\boldsymbol{\mu}^{\prime}}^{\prime} \psi_{\mathfrak{t}_{\boldsymbol{\mu}}}^{\boldsymbol{\alpha}_{k_{\mathfrak{t}}}} \mid \mathfrak{t} \in \operatorname{Std}\left(\boldsymbol{\alpha}_{k}\right) \text { for } 1 \leq k \leq z\right\}
$$

is a basis of $z_{\boldsymbol{\mu} \uparrow i} \mathscr{H}_{n+1}^{\Lambda}$.

Proof. Let $I^{\mu}$ be the vector space spanned by $\mathcal{B}_{\mu, i}$. By [7, Corollary 5.8] and Lemma4.4 we know that

$$
\operatorname{dim} z_{\boldsymbol{\mu} \uparrow i} \mathscr{H}_{n+1}^{\Lambda}=\operatorname{dim} i \text {-Ind } S^{\boldsymbol{\mu}}=\# \mathcal{B}_{\boldsymbol{\mu}, i} \geq \operatorname{dim} I^{\boldsymbol{\mu}} .
$$

Therefore, to prove the Proposition, it suffices to show that $z_{\mu \uparrow i} \mathscr{H}_{n+1}^{\Lambda} \subseteq I^{\mu}$.

For each multipartition $\boldsymbol{\rho}$ of $n$ let $\boldsymbol{\rho}^{+}$be the multipartition of $n+1$ obtained by adding its lowest addable node. Similarly, if $\mathfrak{s} \in \operatorname{Std}(\rho)$ then define $\mathfrak{s}^{+}$to be the unique standard $\boldsymbol{\rho}^{+}$-tableau such that $\mathfrak{s}_{n}^{+}=\mathfrak{s}$. Note that it is not necessarily true that $\operatorname{res}_{\mathfrak{s}^{+}}(n+1)=i$ since the lowest addable node of $\rho$ need not be an $i$-node.

Suppose that $h \in \mathscr{H}_{n+1}^{\Lambda}$. We want to show that $z_{\boldsymbol{\mu} \uparrow i} h \in I^{\mu}$. By definition, $z_{\boldsymbol{\mu} \uparrow i}=$ $e_{i, n} y_{\boldsymbol{\mu}^{\prime}}^{\prime} \psi_{\mathfrak{t}_{\mu} t^{\mu}} e_{i, n}$. Moreover, under the natural embedding $\mathscr{H}_{n}^{\Lambda} \hookrightarrow \mathscr{H}_{n+1}^{\Lambda}$ of ungraded algebras, $m_{\mathfrak{t}_{\mu} \mathfrak{t}^{\mu}}=m_{\mathfrak{t}_{\mu}^{+}\left(\mathfrak{t}^{\mu}\right)^{+}}$. Therefore, applying Theorem 3.7 twice,

$$
\begin{aligned}
& z_{\boldsymbol{\mu} \uparrow i}=e_{i, n} y_{\boldsymbol{\mu}^{\prime}}^{\prime}\left(\sum_{(\mathfrak{s t}) \in \operatorname{Std}^{2}\left(\mathscr{P}_{n+1}\right)} a_{\mathfrak{s t}} \psi_{\mathfrak{s t}}\right) e_{i, n}, \\
& (\mathfrak{s , t}) \geq\left(\mathfrak{t}_{\mu}^{+},\left(\mathfrak{t}^{\mu}\right)^{+}\right)
\end{aligned}
$$


for some $a_{\mathfrak{s t}} \in K$. Therefore, $z_{\boldsymbol{\mu} \uparrow i} h$ is equal to a linear combination of elements of the form $e_{i, n} y_{\boldsymbol{\mu}^{\prime}}^{\prime} \psi_{\mathfrak{s t}}$, where $\mathfrak{s}, \mathfrak{t} \in \operatorname{Std}(\boldsymbol{\lambda}), \operatorname{res}_{\mathfrak{s}}(n+1)=i$ and $\boldsymbol{\lambda}$ is a multipartition $n+1$ such that $\boldsymbol{\lambda} \unrhd \boldsymbol{\mu}^{+}$. In particular, Shape $\left(\mathfrak{s}_{n}\right) \unrhd \boldsymbol{\mu}$.

On the other hand, since $e_{\boldsymbol{\mu}^{\prime}}^{\prime} y_{\boldsymbol{\mu}^{\prime}}^{\prime}=\psi_{\mathbf{t}^{\mu^{\prime}} \boldsymbol{t}^{\mu^{\prime}}}^{\prime}$ and $e_{i, n} \in K\left[y_{1}, \cdots, y_{n+1}\right]$, applying Theorem 3.7 and Corollary 3.9 shows that $e_{i, n} e_{\boldsymbol{\mu}^{\prime}}^{\prime} y_{\boldsymbol{\mu}^{\prime}}^{\prime}$ is equal to a linear combination of elements $\psi_{\mathfrak{u v}}^{\prime}$ such that $\mathfrak{u} \unrhd\left(\mathfrak{t}^{\boldsymbol{\mu}^{\prime}}\right)^{+}$and $\mathfrak{v} \unrhd\left(\mathfrak{t}^{\boldsymbol{\mu}^{\prime}}\right)^{+}$. By Corollary 3.8, $\psi_{\mathfrak{u v}}^{\prime} \psi_{\mathfrak{s t}} \neq 0$ only if $\mathfrak{v}^{\prime} \unrhd \mathfrak{s}$. Therefore, $e_{i, n} e_{\boldsymbol{\mu}^{\prime}}^{\prime} y_{\boldsymbol{\mu}^{\prime}}^{\prime} \psi_{\mathfrak{s t}} \neq 0$ only if $\mathfrak{t}_{\boldsymbol{\mu}} \unrhd \mathfrak{s}_{n}$ because $\mathfrak{t}_{\boldsymbol{\mu}}=\left(\mathfrak{t}^{\boldsymbol{\mu}^{\prime}}\right)^{\prime} \unrhd\left(\mathfrak{v}^{\prime}\right)_{n}$. Therefore, by the last paragraph, $z_{\boldsymbol{\mu} \uparrow i} h=e_{i, n} y_{\boldsymbol{\mu}^{\prime}}^{\prime} \psi_{\mathfrak{t}_{\mu} t^{\mu}} e_{i, n} h=e_{i, n} e_{\boldsymbol{\mu}^{\prime}}^{\prime} y_{\boldsymbol{\mu}^{\prime}}^{\prime} \psi_{\mathfrak{t}_{\mu} \mathfrak{t}^{\mu}} e_{i, n} h$ is equal to a linear combination of the elements of the form $e_{i, n} y_{\boldsymbol{\mu}^{\prime}}^{\prime} \psi_{\mathfrak{s t}}$, where $\mathfrak{s}, \mathfrak{t} \in \operatorname{Std}(\boldsymbol{\lambda})$, $\operatorname{Shape}\left(\mathfrak{s}_{n}\right) \unrhd \boldsymbol{\mu}, \operatorname{res}_{\mathfrak{s}}(n+1)=i$, and $\boldsymbol{\lambda}$ is a multipartition of $n+1$ such that $\mathfrak{t}_{\boldsymbol{\mu}} \unrhd \mathfrak{s}_{n}$. It follows that $\mathfrak{s}=\mathfrak{t}_{\boldsymbol{\mu}}^{\boldsymbol{\alpha}_{j}}$ and $\boldsymbol{\lambda}=\boldsymbol{\alpha}_{j}$, for some $j$ with $1 \leq j \leq z$. Hence, $z_{\boldsymbol{\mu} \uparrow i} h \in I^{\mu}$ and the proof is complete.

We can now prove our main theorem.

Proof of Main Theorem. By Lemma 4.4 $i$-Ind $S^{\boldsymbol{\mu}}\left\langle\operatorname{def} \beta+\operatorname{codeg} \mathfrak{t}_{\boldsymbol{\mu}}\right\rangle \cong z_{\boldsymbol{\mu} \uparrow i} \mathscr{H}_{n+1}^{\Lambda}$ so it remains to show that $z_{\mu \uparrow i} \mathscr{H}_{n+1}^{\Lambda}$ has a suitable Specht filtration. For $0 \leq j \leq n$ define

$$
\left.I_{j}=\left\langle e_{i, n} y_{\boldsymbol{\mu}^{\prime}}^{\prime} \psi_{\mathfrak{t}_{\mu} \alpha_{k}}^{\alpha_{k}}\right| \mathfrak{t} \in \operatorname{Std}\left(\boldsymbol{\alpha}_{k}\right) \text { for } 1 \leq k \leq j\right\rangle
$$

Then $I_{j}$ is a submodule of $z_{\boldsymbol{\mu} \uparrow i} \mathscr{H}_{n+1}^{\Lambda}$ by Theorem 3.5 and the proof of Proposition 4.5 Furthermore, the map

$$
S^{\boldsymbol{\alpha}_{j}}\left\langle 2 \operatorname{codeg} \mathfrak{t}_{\boldsymbol{\mu}}+\operatorname{deg} \mathfrak{t}_{\boldsymbol{\mu}}^{\boldsymbol{\alpha}_{j}}\right\rangle \cong I_{j} / I_{j-1} ; \psi_{\mathfrak{t}} \mapsto e_{i, n} y_{\boldsymbol{\mu}^{\prime}}^{\prime} \psi_{\mathbf{t}_{\mu}}^{\boldsymbol{\alpha}_{j}}{ }^{\boldsymbol{t}_{1}}+I_{j-1},
$$

for $\mathfrak{t} \in \operatorname{Std}\left(\alpha_{j}\right)$ is an isomorphism since $\mathcal{B}_{\boldsymbol{\mu}, i}$ is linearly independent. Notice that this map can also be viewed as left multiplication:

$$
\psi_{\mathbf{t}_{\mu} \boldsymbol{\alpha}_{j}}+\mathscr{H}_{\boldsymbol{\mu}} \mapsto e_{i, n} y_{\boldsymbol{\mu}^{\prime}}^{\prime} \psi_{\mathbf{t}_{\mu^{\prime}} \boldsymbol{\alpha}_{\mathbf{t}}}+I_{j-1}
$$

for $\mathfrak{t} \in \operatorname{Std}\left(\boldsymbol{\alpha}_{j}\right)$. To complete the proof we need to check that the degree shifts in the Specht filtration are as predicted by the Main Theorem. We have $\operatorname{deg} \mathfrak{t}_{\boldsymbol{\mu}}^{\boldsymbol{\alpha}_{j}}=\operatorname{deg} \mathfrak{t}_{\boldsymbol{\mu}}+$ $d_{A_{j}}(\boldsymbol{\mu})$. Moreover, codeg $\mathfrak{t}_{\boldsymbol{\mu}}+\operatorname{deg} \mathfrak{t}_{\boldsymbol{\mu}}=\operatorname{def} \beta$ by [8, Lemma 3.12]. Therefore,

$$
2 \operatorname{codeg} \mathfrak{t}_{\boldsymbol{\mu}}+\operatorname{deg} \mathfrak{t}_{\boldsymbol{\mu}}^{\boldsymbol{\alpha}_{j}}=\operatorname{def} \beta+\operatorname{codeg} \mathfrak{t}_{\boldsymbol{\mu}}+d_{A_{j}}(\boldsymbol{\mu}),
$$

so that $S^{\boldsymbol{\alpha}_{j}}\left\langle\operatorname{def} \beta+\operatorname{codeg} \mathfrak{t}_{\boldsymbol{\mu}}+d_{A_{j}}(\boldsymbol{\mu})\right\rangle \cong I_{j} / I_{j-1}$. Applying Lemma4.4 now completes the proof of our Main Theorem.

Finally, we note that we also have the following description of the induced graded dual Specht module. This can be proved either using the isomorphism $S^{\boldsymbol{\mu}} \cong S_{\boldsymbol{\mu}^{\prime}}^{\circledast}\langle\operatorname{def} \beta\rangle$ from [13. Prop. 6.19], or by essentially repeating the argument above starting with the isomorphism $S_{\boldsymbol{\mu}^{\prime}}\left\langle\operatorname{def} \beta+\operatorname{deg} \boldsymbol{t}^{\boldsymbol{\mu}}\right\rangle \cong z_{\boldsymbol{\mu}^{\star}} \mathscr{H}_{n}^{\Lambda}$ from Lemma 4.2. We leave the details to the reader.

4.6. Corollary. Suppose that $\boldsymbol{\mu} \in \mathscr{P}_{n}$ and $i \in I$. Then $i$-Ind $S_{\boldsymbol{\mu}^{\prime}}$ has a filtration

$$
0=J_{z+1} \subset J_{z} \subset \cdots \subset J_{1}=i \text {-Ind } S_{\boldsymbol{\mu}^{\prime}},
$$

such that $J_{k} / J_{k+1} \cong S_{\boldsymbol{\alpha}_{k}}\left\langle d^{A_{k}}(\boldsymbol{\mu})\right\rangle$, for $1 \leq k \leq z$, where $\left\{A_{1}<A_{2}<\cdots<A_{z}\right\}$ is the set of addable $i$-nodes of $\boldsymbol{\mu}^{\prime}$ ordered so that $\boldsymbol{\alpha}_{z} \triangleright \cdots \triangleright \boldsymbol{\alpha}_{1}$, where $\boldsymbol{\alpha}_{k}=\boldsymbol{\mu}^{\prime} \cup\left\{A_{k}\right\}$ for $1 \leq k \leq z$. 


\section{REFERENCES}

[1] S. ARIKI, On the decomposition numbers of the Hecke algebra of $G(m, 1, n)$, J. Math. Kyoto Univ., 36 (1996), 789-808.

[2] S. ARIKI AND K. KOIKE, A Hecke algebra of $(\mathbf{Z} / r \mathbf{Z})$ ? $\mathfrak{S}_{n}$ and construction of its irreducible representations, Adv. Math., 106 (1994), 216-243.

[3] S. Ariki, A. Mathas, And H. Rui, Cyclotomic Nazarov-Wenzl algebras, Nagoya J. Math., 182 (2006), 47-134. (Special issue in honour of George Lusztig).

[4] J. BRundan, Centers of degenerate cyclotomic Hecke algebras and parabolic category $\mathcal{O}$, Represent. Theory, 12 (2008), 236-259.

[5] J. BRUndan AND A. KLeshchev, Schur-Weyl duality for higher levels, Selecta Math., 14 (2008), 1-57.

[6] —, Blocks of cyclotomic Hecke algebras and Khovanov-Lauda algebras, Invent. Math., 178 (2009), $451-484$.

[7] — Graded decomposition numbers for cyclotomic Hecke algebras, Adv. Math., 222 (2009), 18831942.

[8] J. Brundan, A. Kleshchev, AND W. WANG, Graded Specht modules, preprint, 2009. arXiv:math.RT/0901.0218.

[9] J. BRUNDAN AND C. STROPPEL, Highest weight categories arising from Khovanov's diagram algebra III: category $O,(2008)$, preprint. arXiv.RT/arXiv:0812.1090.

[10] R. Dipper, G. James, AND A. Mathas, Cyclotomic q-Schur algebras, Math. Z., 229 (1999), 385-416.

[11] R. Dipper And A. Mathas, Morita equivalences of Ariki-Koike algebras, Math. Zeit., 240 (2002), 579610.

[12] J. J. Graham And G. I. Lehrer, Cellular algebras, Invent. Math., 123 (1996), 1-34.

[13] J. Hu AND A. Mathas, Graded cellular bases for the cyclotomic Khovanov-Lauda-Rouquier algebras of type A, Adv. Math., to appear. arXiv:0907.2985 (46 pages).

[14] G. D. James AND A. Mathas, The Jantzen sum formula for cyclotomic q-Schur algebras, Trans. Amer. Math. Soc., 352 (2000), 5381-5404.

[15] V. G. KAC, Infinite dimensional Lie algebras, CUP, Cambridge, third ed., 1994.

[16] M. Khovanov AND A. D. LAUdA, A diagrammatic approach to categorification of quantum groups. I, Represent. Theory, 13 (2009), 309-347.

[17] S. LYLE AND A. MATHAS, Blocks of cyclotomic Hecke algebras, Adv. Math., 216 (2007), 854-878.

[18] A. MATHAS, Hecke algebras and Schur algebras of the symmetric group, Univ. Lecture Notes, 15, Amer. Math. Soc., 1999.

[19] — Tilting modules for cyclotomic Schur algebras, J. Reine Angew. Math., 562 (2003), 137-169.

[20] - Matrix units and generic degrees for the Ariki-Koike algebras, J. Algebra, 281 (2004), 695-730.

[21] — Seminormal forms and Gram determinants for cellular algebras, J. Reine Angew. Math., 619 (2008), 141-173. With an appendix by M. Soriano.

[22] - A Specht filtration of an induced Specht module, J. Algebra, 322 (2009), 893-902.

[23] G. E. MURPHY, The idempotents of the symmetric group and Nakayama's conjecture, J. Algebra, 81 (1983), 258-265.

[24] - The representations of Hecke algebras of type $\mathbf{A}_{\mathbf{n}}, \mathrm{J}$. Algebra, 173 (1995), 97-121.

[25] R. RouQUIER, 2-Kac-Moody algebras, preprint 2008. arXiv:0812.5023

[26] S. RYOM-HANSEN, Grading the translation functors in type A, J. Algebra, 274 (2004), 138-163.

School of Mathematics and Statistics F07, University of Sydney, NSW 2006, Australia E-mail address: junhu303@yahoo.com.cn

School of Mathematics and Statistics F07, University of Sydney, NSW 2006, Australia

E-mail address: a . mathas@usyd.edu. au 